\title{
Topological Quantization and Cohomology
}

\author{
Orlando Alvarez ${ }^{\star}$ \\ Department of Physics and Lawrence Berkeley Laboratory, University of California, Berkeley, \\ CA 94720 , USA
}

\begin{abstract}
The relationships between topological charge quantization, Lagrangians and various cohomology theories are studied. A very general criterion for charge quantization is developed and applied to various physical models. The relationship between cohomology and homotopy is discussed.
\end{abstract}

\section{Introduction}

Topology and geometry have been playing an important role in our current theoretical understanding of quantum field theories. One of the most interesting applications of topology has been the quantization of certain coupling constants. In this article we present a very general framework under which one can understand coupling constant quantization. Firstly, to argue that the correct framework for analyzing topological quantization is the mathematical discipline known as Cech cohomology. Secondly, to develop the ideas of Cech cohomology by using familiar examples from physics.

We will see that Dirac's magnetic charge quantization condition [1], the quantization of the coupling constant [2] in the Wess-Zumino Lagrangian [3], the quantization [4] of the mass term in three dimensional Yang-Mills theory [5], and the Bagger-Witten gravitational constant quantization [6] can all be formulated within the same framework.

The mathematical formalism we will discuss is standard mathematics [7] which is unfamiliar to most physicists. In this paper we stress the relationship of this abstract mathematical formalism to the Lagrangian mechanics of physicists. We will see that the origin of the quantization conditions is closely related to a famous theorem from classical mechanics that states that two Lagrangians which differ by a total derivative lead to equivalent dynamics. Generalizing some ideas of $\mathrm{Wu}$ and

* This work was supported in part by the National Science Foundation under Contracts PHY 81-18547; and by the Director, Office of High Energy and Nuclear Physics of the U.S. Department of Energy under Contracts DE-AC03-76SF00098

$\star \star$ Alfred P. Sloan Foundation Fellow 
Yang [8] we will show how certain quantization conditions arise in arbitrary dimensional manifolds. We will show that it is not very difficult to obtain a quantization condition, and that quantization conditions do not have to arise from group theoretic considerations. All our considerations will exploit well established results in cohomology theory.

Many of the results of this article have been previously derived by using other methods, mostly homotopy theory. In this article we will contrast homotopy with cohomology theory. We will see that there are situations where the homotopy arguments cannot be used but the cohomology arguments are applicable. If the homotopy arguments are applicable then the cohomology arguments are also applicable but the converse is not true.

We will not be explicit as to whether we are in Euclidean space or in Minkowski space. The topological term is always chosen such that it contributes a complex phase to the path integral.

This paper is organized in the following way. In Sect. 2 we discuss the familiar Dirac quantization condition by modifying some ideas of $\mathrm{Wu}$ and Yang [8]. In Sect. 3 we generalize the ideas of $\mathrm{Wu}$ and Yang to two dimensional field theories. In Sect. 4 we introduce Cech cohomology and demonstrate that the ideas presented in Sects. 2 and 3 can be incorporated into this framework. In Sect. 5 we return to physics. We finish the article by contrasting homotopy and cohomology in Sect. 6 .

\section{Dirac's Quantization Condition Revisited}

In this section, the familiar Dirac quantization condition is derived in a manner illustrating Cech cohomological concepts. We will use a generalization of some ideas of $\mathrm{Wu}$ and Yang [8]. The methods of this section extend to higher dimensional cases.

Consider the motion of a point particle on a two dimensional sphere with a magnetic monopole residing at the center of the sphere. The classical Lagrangian for this system is

$$
\mathrm{L}=\frac{1}{2}\left(\frac{d x}{d t}\right)^{2}+\frac{1}{4 e^{2}} F_{\mu \nu} F^{\mu \nu}+A_{\mu} \frac{d x^{\mu}}{d t} .
$$

The term of interest for us is the coupling of the vector potential to the velocity of the particle. This is the only term of topological interest and for the remaining part of this section we will completely disregard the kinetic energy terms. We would like to view this term as the line integral of the one form $A=A_{\mu} d x^{\mu}$ along the trajectory $\Gamma$ of the particle:

$$
\int_{\Gamma} A
$$

This would be fine except for the fact that it is impossible to find an everywhere non-singular vector potential over the entire sphere. Wu and Yang [8] pointed out how to modify the Lagrangian to take this into account. Cover the sphere with a collection of open sets $\mathscr{U}=\left\{U_{\alpha}\right\}$. On each open set choose a vector potential one form $A_{\alpha}$. The subscript $\alpha$ on $A_{\alpha}$ is not a Lorentz index and refers to the open cover: 


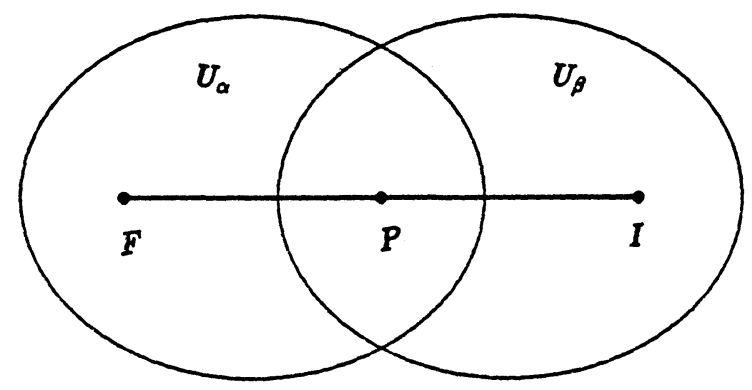

Fig. 2.1. The worldline of the particle which begins at $I$ and ends at $F$ traverses two distinct coordinate patches. The point $P$ is in the intersection of the two patches

$A_{\alpha}=A_{\alpha \mu} d x^{\mu}$. Consider the situation depicted in Fig. 2.1 where one has a trajectory $\Gamma$ that goes through a non-empty overlap $U_{\alpha} \cap U_{\beta}$. Let $P$ be a point in the intersection. Naively one would write the vector potential contribution to the action as (remember that we are concentrating only on the term of possible topological interest)

$$
I_{P}=\int_{P}^{F} A_{\alpha}+\int_{I}^{P} A_{\beta} .
$$

The problem with this definition is that it depends on the choice of the point $P$. To see this, consider another point $Q$ in the overlap, construct $I_{Q}$ and compute the difference $I_{Q}-I_{P}$ :

$$
I_{Q}-I_{P}=-\int_{P}^{Q}\left(A_{\alpha}-A_{\beta}\right) .
$$

We require knowledge of the gauge transformation on the overlap to evaluate Eq. (2.4). On each overlap it is necessary to specify a gauge transformation $\psi_{\alpha \beta}$ satisfying

$$
d \psi_{\alpha \beta}=A_{\alpha}-A_{\beta} .
$$

Note that $-\psi_{\alpha \beta}=\psi_{\beta \alpha}$. Equation (2.4) is entirely determined by the gauge transformation:

$$
I_{Q}-I_{P}=\psi_{\alpha \beta}(P)-\psi_{\alpha \beta}(Q) .
$$

In particular the quantity $I=I_{Q}+\psi_{\alpha \beta}(Q)$ is independent of the choice of point $Q$. More explicitly, $I$ is given by

$$
I=\int_{Q}^{F} A_{\alpha}+\psi_{\alpha \beta}(Q)+\int_{I}^{Q} A_{\beta} .
$$

This is the correct form of the action which was given by $\mathrm{Wu}$ and Yang. It seems to be a bit mysterious but its significance is more discernible by thinking about quantum mechanics. According to the Feynman path integral formulation of quantum mechanics [9], the effect of a vector potential on propagation is to multiply the amplitudes by the exponential of Eq. (2.7). This is simply seen to be the amplitude for propagation in patch $U_{\beta}$, followed by a gauge transformation and terminating with the amplitude to propagate in the new gauge in patch $U_{\alpha}$. 


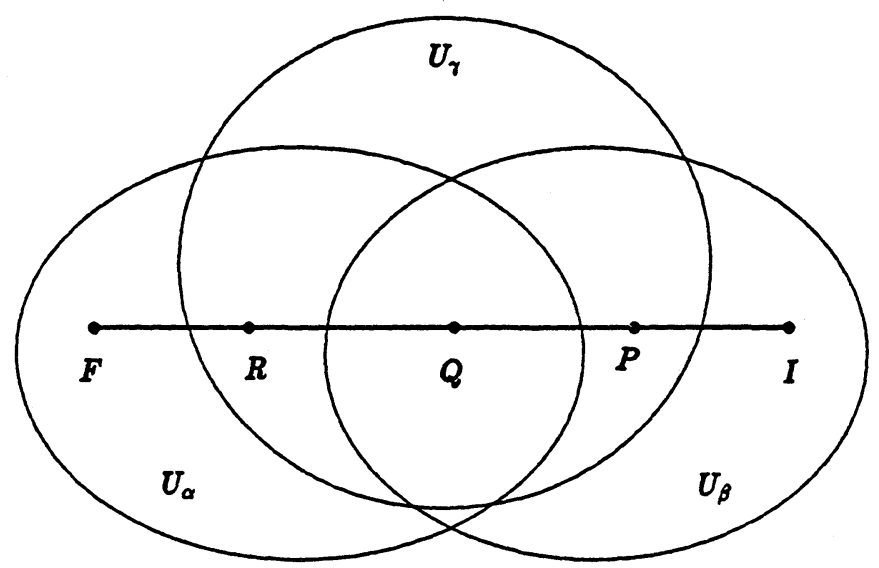

Fig. 2.2. A third coordinate patch is introduced. The points $P$ and $R$ do not have to be in the triple intersection

We now depart from the discussion of Wu and Yang and we ask the question, "What happens in a triple overlap?". The situation is depicted in Fig. 2.2. Let us temporarily forget $U_{\gamma}$. The action is given by Eq. (2.7). Remember that the value of the action is independent of the location of $Q$. Let us rewrite this term in such a way that contribution to the line integral from the part of the trajectory between $P$ and $R$ is expressed in terms of $A_{\gamma}$ only. By using the gauge transformation law for the vector potential the action may be rewritten as:

$$
\begin{aligned}
I= & \int_{R}^{F} A_{\alpha}+\psi_{\alpha \gamma}(R)+\int_{P}^{R} A_{\gamma}+\psi_{\gamma \beta}(P)+\int_{I}^{P} A_{\beta} \\
& +\left(\psi_{\alpha \beta}(Q)+\psi_{\beta \gamma}(Q)+\psi_{\gamma \alpha}(Q)\right) .
\end{aligned}
$$

This equation is reminiscent of the Wu-Yang prescription. It is of the form line integral, gauge transformation, line integral, gauge transformation, line integral, and a left-over piece. It is important to note that the left-over piece contains the only reference to the point $Q$. The other pieces are just the $\mathrm{Wu}$-Yang prescription for going from patch $U_{\beta}$ to patch $U_{\gamma}$ and ending in patch $U_{\alpha}$. We will see that the left-over piece contains all the information required to obtain Dirac's quantization condition.

The first piece of information we need is that the gauge transformations must satisfy a consistency condition on triple overlaps. Consider the following three equations:

$$
\begin{aligned}
& A_{\alpha}-A_{\beta}=d \psi_{\alpha \beta}, \\
& A_{\beta}-A_{\gamma}=d \psi_{\beta \gamma}, \\
& A_{\gamma}-A_{\alpha}=d \psi_{\gamma \alpha} .
\end{aligned}
$$

Add all three equations to obtain the result

$$
d\left(\psi_{\alpha \beta}+\psi_{\beta \gamma}+\psi_{\gamma \alpha}\right)=0
$$

To proceed further we need a special condition on the cover we chose for the sphere. It is possible to choose a cover such that each $U_{\delta}$ is diffeomorphic to an open ball, and each non-empty finite multiple intersection is also diffeomorphic to 
an open ball [7]. This means that the Poincare lemma is valid in each multiple intersection. In particular, we reach the conclusion that on $U_{\alpha} \cap U_{\beta} \cap U_{\gamma}$ one has

$$
\psi_{\alpha \beta}+\psi_{\beta \gamma}+\psi_{\gamma \alpha}=c_{\alpha \beta \gamma},
$$

where $c_{\alpha \beta \gamma}$ is a constant over the entire triple overlap. Therefore Eq. (2.8) is independent of $Q$ as required.

There is an important lesson that this exercise teaches us. The classical action is ambiguous up to a constant. A priori, one could use the $\mathrm{Wu}$-Yang prescription to write an expression involving patches $U_{\alpha}$ and $U_{\beta}$ only, or write an expression involving patches $U_{\alpha}, U_{\beta}, U_{\gamma}$. The difference between these two expressions is a constant which does not affect the classical equations of motion.

This classical ambiguity leads to quantum mechanical inconsistencies unless certain conditions are imposed on the collection of all $\left\{c_{\alpha \beta \gamma}\right\}$. The best way to see this is through path integral quantization. Consider the contribution of a trajectory $\Gamma$ to the non-relativistic propagator:

$$
\exp \left(\underset{\Gamma}{i \int_{\Gamma} A}\right) \cdot K_{\text {free }}(\Gamma) \text {. }
$$

The only ambiguity arises in how one decides to evaluate the vector potential line integral. There is an ambiguous phase factor of $\exp \left(i c_{\alpha \beta \gamma}\right)$. Such a potential ambiguity exists at each non-empty triple intersection of patches on the sphere. The only way to avoid this mishap is to require that each phase factor be equal to one. In other words one has to choose all $c_{\alpha \beta \gamma}$ to be $2 \pi \times$ (integer). Later we will see that this statement contains topological information about the manifold. It states that if the manifold's second cohomology class contains the integers then a consistent quantum theory requires an appropriate coupling constant to be quantized. In fact, the collection $\left\{c_{\alpha \beta \gamma}\right\}$ defines a two cocycle.

We now turn to the relationship of the $\left\{c_{\alpha \beta \gamma}\right\}$ to Dirac's charge quantization condition. Assume we integrate the magnetic field over the sphere. We would like to write this surface integral as a sum of integrals over each of the coordinate patches:

$$
\int_{S^{2}} F=\sum_{\alpha} \int_{U_{\alpha}} F
$$

This is not correct since one has overcounted contributions from the overlaps. Let us subdivide the manifold into regions $V_{\alpha}$ as depicted in Fig. 2.3. Since on each $V_{\alpha}$

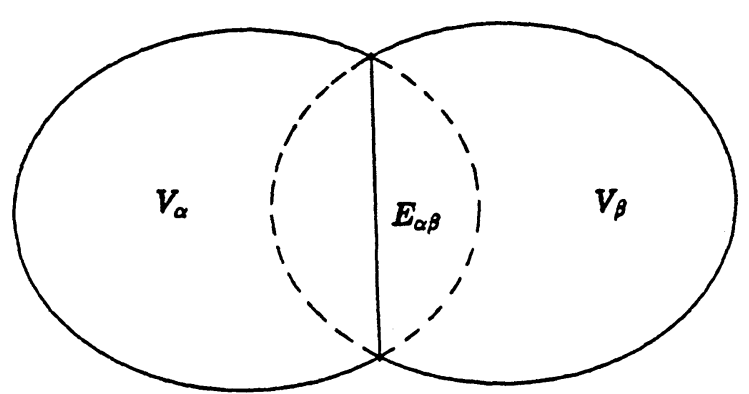

Fig. 2.3. The subdivision of the patches $U_{\alpha}$ and $U_{\beta}$ into non-overlapping subsets $V_{\alpha}$ and $V_{\beta}$ 


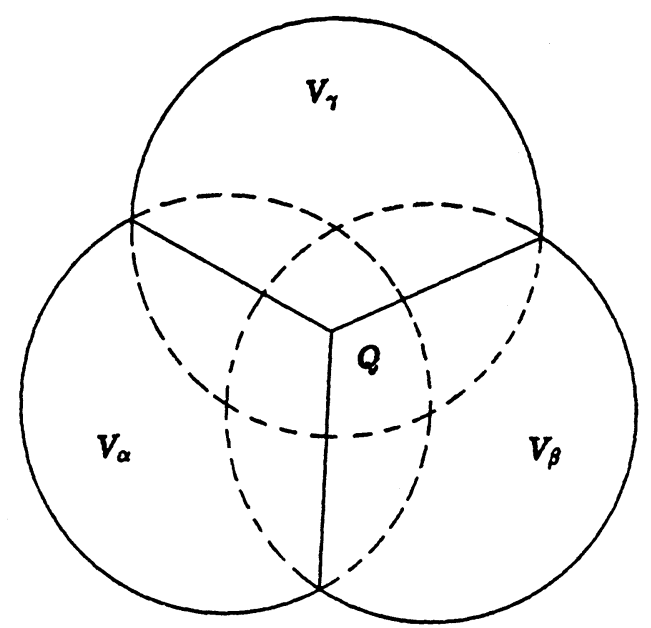

Fig. 2.4. The subdivision of a triple region into three non-overlapping subsets. Note that a preferred point $Q$ is introduced at the junction

the potential $A_{\alpha}$ is well defined, we are allowed to use Stokes' theorem and perform the following manipulations:

$$
\int_{V_{\alpha}} F=\int_{V_{\alpha}} d A_{\alpha}=\int_{\vartheta V_{\alpha}} A_{\alpha}
$$

Along the common border of $V_{\alpha}$ and $V_{\beta}$ there is a contribution to the total magnetic flux through these two patches which may be expressed as the line integral of the difference of the two vector potentials along the edge $E_{\alpha \beta}$, see Fig. 2.3. One can now use the gauge transformation $\psi_{\alpha \beta}$ and Stokes' theorem to conclude that

$$
\int_{E_{\alpha \beta}}\left(A_{\alpha}-A_{\beta}\right)=\int_{\vartheta E_{\alpha \beta}} \psi_{\alpha \beta} .
$$

This contribution only depends on the value of the gauge transformation at the endpoints of $E_{\alpha \beta}$. We are not finished because we have to worry about what happens at triple intersections, see Fig. 2.4. Note that there is a contribution to the total flux through the three patches which is given by

$$
\psi_{\alpha \beta}(Q)+\psi_{\beta \gamma}(Q)+\psi_{\gamma \alpha}(Q) \text {. }
$$

Each $\psi$ arises from the corresponding edge. With a little work one can see that the total magnetic flux is given by

$$
\int_{S^{2}} F=\sum_{V_{\alpha \beta \gamma}} c_{\alpha \beta \gamma}
$$

where the sum is over all triple overlaps with $V_{\alpha \beta \gamma}=U_{\alpha} \cap U_{\beta} \cap U_{\gamma} \cap S^{2}$. We conclude that the total flux is given by

$$
\int_{S^{2}} F=2 \pi \sum_{V_{\alpha \beta \gamma}} n_{\alpha \beta \gamma}
$$

where the integer $n$ is given by

$$
c_{\alpha \beta \gamma}=2 \pi n_{\alpha \beta \gamma} .
$$


This is Dirac's quantization condition. We shall see that this condition generalizes in higher dimensions. Note that the quantization arose because of consistency conditions on triple overlaps of the different coordinate patches. There is a connection between the ambiguity in the classical action and the total flux through the sphere. In the case of a sphere, the construction given above is not necessary since one can cover the sphere with two coordinate patches, see $\mathrm{Wu}$ and Yang [8]. The above construction is valid for any manifold.

In this example, one finds that no further conditions are imposed by looking at quadruple or higher overlaps. This is not true in two dimensional field theories as we will see in the next section.

\section{A Field Theory Example}

In this section we generalize the construction of $\mathrm{Wu}$ and Yang to the field theory case. We will see that there is a topological quantization that occurs which is closely related to the quantization of the Wess-Zumino term. Assume that spacetime is a manifold $S$ of dimension two with no spatial boundary. Possible examples are $S^{2}, \mathbb{R} \times S^{1}$, or $T^{2}=S^{1} \times S^{1}$. The classical field is given by a map

$$
\phi: S \rightarrow M \text {. }
$$

The manifold $M$ can be very general. We are just working with the generalized nonlinear sigma model. There will be various contributions to the Lagrangian but we will study only a very special type of term. Assume that there is a contribution to the Lagrangian which can be associated with a two form on M. More explicitly, assume that there is a term in the Lagrangian of the form

$$
\int_{S} f_{a b}(\phi) \frac{\partial \phi^{a} \partial \phi^{b}}{\partial x^{\mu} \partial x^{\nu}} d x^{\mu} \wedge d x^{\nu}
$$

The contribution to the action may be rewritten as

$$
\int_{\phi(S)} f_{a b}(\phi) d \phi^{a} \wedge d \phi^{b}
$$

where $\phi(S)$ is just the image of spacetime $S$ under the field $\phi$, and where $T$ is given by

$$
T=f_{a b}(\phi) d \phi^{a} \wedge d \phi^{b} .
$$

This is analogous to what we did with the line integral of the vector potential in the previous section. For our purposes one should always think of such a contribution to the Lagrangian as being a differential form on the field manifold $M$ and not as a differential form on the spacetime manifold $S$.

The situation is more complicated. In general the differential two form $T$ is not globally defined on $M$. This means that one is going to have to subdivide the image of spacetime $\phi(S)$ into distinct patches just as in the electromagnetic case. We will do better by subdividing all of $M$ into patches by choosing an open cover $\mathscr{U}=\left\{U_{\alpha}\right\}$ of $M$. A mathematical theorem [7] says that we can choose the cover in such a way that every non-empty finite intersection is diffeomorphic to an open ball in $\mathbb{R}^{n}$ where $n$ is the dimension of $M$. Assume that there is a collection of locally defined differential two forms $\left\{T_{\alpha}\right\}$, where $T_{\alpha}$ is the Lagrangian one has to use in patch $U_{\alpha}$. 


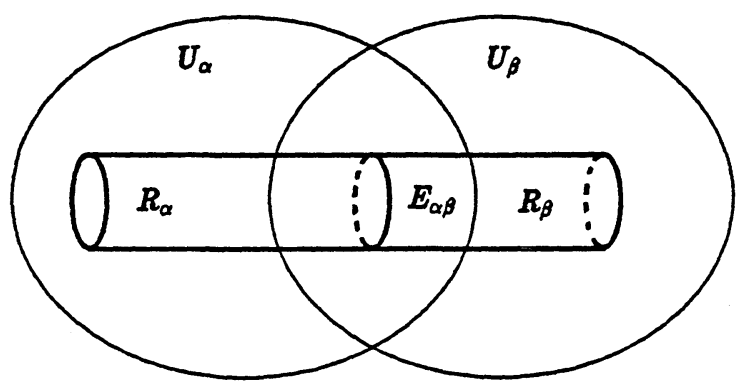

Fig. 3.1. The evolution of a manifold with its spatial topology being a circle. The world sheet lies in two distinct patches. It is subdivided into regions $R_{\alpha}$ and $R_{\beta}$ with the edge being $E_{\alpha \beta}$

Thus far we are mimicking the electromagnetic situation. The key to a future quantization condition is the following assumption. On a non-empty overlap $U_{\alpha}$ $\cap U_{\beta}$ one assumes that the respective Lagrangians differ by the differential of a one form $J_{\alpha \beta}$

$$
T_{\alpha}-T_{\beta}=d J_{\alpha \beta}
$$

We will see that such a strong restriction imposes conditions on the manifold $M$.

The standard Wess-Zumino term is actually of this type. The transformation properties of the Wess-Zumino term may be found in [10]. Remember that we have chosen a cover of the manifold in such a way that the Poincare lemma is always valid locally. Such covers are not usually chosen by physicists and one has to worry about global topological considerations, see for example $\mathrm{Wu}$ and Yang [8].

It is possible to generalize the Wu-Yang construction to this situation. For simplicity, let us assume that the image of spacetime $\phi(S)$ lies entirely in the patches $U_{\alpha}$ and $U_{\beta}$ as depicted in Fig. 3.1. By mimicking the construction of Sect. 1 one can show that

$$
\int_{R_{\alpha}} T_{\alpha}-\int_{E_{\alpha \beta}} J_{\alpha \beta}+\int_{R_{\beta}} T_{\beta},
$$

is independent of where one chooses the boundary $E_{\alpha \beta}$. This is the generalization of Eq. (2.7). This prescription is actually incomplete. We will have to do a further modification to reach a satisfactory answer within the domain of classical field theory.

We have to worry about what happens in triple overlaps. The situation is depicted in Fig. 3.2. We will see that the introduction of the triple overlap introduces some $E_{\alpha \beta}$ dependence and a modification of Eq. (3.6) is required. The modification is obtained by applying the ideas of Wu and Yang one more time. By using the conditions on the overlaps one can rewrite Eq. (3.6) as

$$
\int_{\boldsymbol{R}_{\alpha}} T_{\alpha}-\int_{E_{\alpha \gamma}} J_{\alpha \gamma}+\int_{\boldsymbol{R}_{\gamma}} T_{\gamma}-\int_{E_{\gamma \beta}} J_{\gamma \beta}+\int_{\boldsymbol{R}_{\gamma}} T_{\beta}-\int_{E_{\alpha \beta}}\left(J_{\alpha \beta}+J_{\beta \gamma}+J_{\gamma \alpha}\right) .
$$

The form of the above is reminiscent of Eq. (2.8). There is an ambiguity in the classical action when one looks at triple overlaps. The above appears to depend on $E_{\alpha \beta}$. In Sect. 1 we found that the ambiguity was a constant. In the present case we 


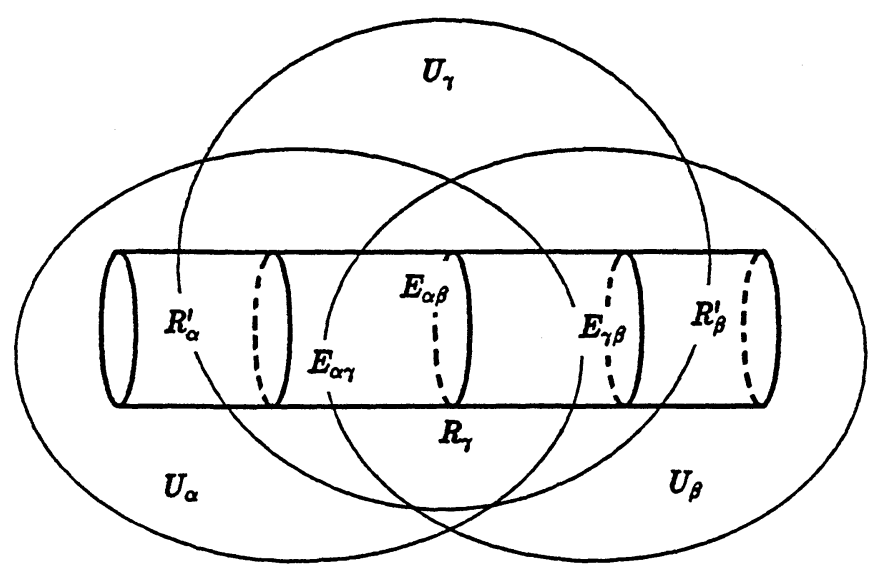

Fig. 3.2. The generalization of Fig. 2.2 to one higher dimension. Note that $R_{\alpha}$ and $R_{\beta}$ have been subdivided into three regions $R_{\alpha}^{\prime}, R_{\beta}^{\prime}$, and $R_{\gamma}$. Also note the new edges

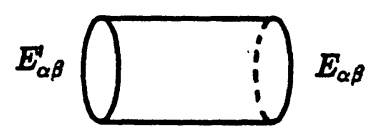

Fig. 3.3. Integration region for studying the dependence of the action on the choice of edge

will have to work a little harder. Consider the following three equations:

$$
\begin{aligned}
& T_{\alpha}-T_{\beta}=d J_{\alpha \beta}, \\
& T_{\beta}-T_{\gamma}=d J_{\beta \gamma}, \\
& T_{\gamma}-T_{\alpha}=d J_{\gamma \alpha} .
\end{aligned}
$$

By adding all three equations we conclude that

$$
d\left(J_{\alpha \beta}+J_{\beta \gamma}+J_{\gamma \alpha}\right)=0 .
$$

This is enough information to verify the independence of the action on $E_{\alpha \beta}$. Consider a nearby loop $E_{\alpha \beta}^{\prime}$. It is clear from Fig. 3.3 that

$$
\int_{E_{\alpha \beta}}\left(J_{\alpha \beta}+J_{\beta \gamma}+J_{\gamma \alpha}\right)=\int_{E_{\alpha \beta}^{\prime}}\left(J_{\alpha \beta}+J_{\beta \gamma}+J_{\gamma \alpha}\right) \text {. }
$$

In the above we used the information contained in Eq. (3.9) and Stokes' theorem.

Since the Poincare lemma is valid in the triple overlaps there exist distinct functions $K_{\alpha \beta \gamma}$ such that

$$
d K_{\alpha \beta \gamma}=J_{\alpha \beta}+J_{\beta \gamma}+J_{\gamma \alpha}
$$

on the respective overlaps. The term involving $E_{\alpha \beta}$ may be rewritten as

$$
\int_{E_{\alpha \beta}} d K_{\alpha \beta \gamma}=\int_{\vartheta E_{\alpha \beta}} K_{\alpha \beta \gamma}=0
$$

This vanishes since $E_{\alpha \beta}$ is boundaryless. 


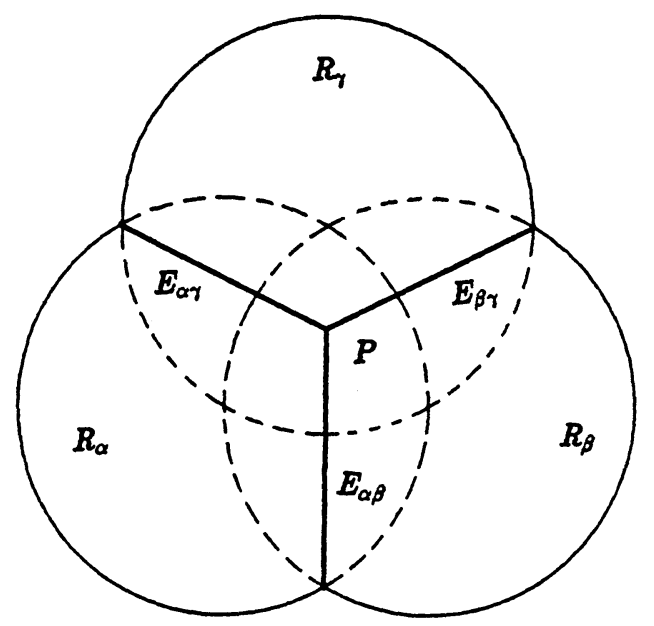

Fig. 3.4. The appearance of Y-junctions when one subdivides a two dimensional integration region into three distinct non-overlapping sets

The triple overlaps analogous to the one dimensional case introduce no ambiguities into the two dimensional field theory. This is unlike the electromagnetic example of $\mathrm{Wu}$ and Yang. We will see that a more careful analysis of triple overlaps requires some modifications of the Wu-Yang procedure. We must analyze a new feature of two dimensional field theories which is not present in the one dimensional example. A suitable modification of the $\mathrm{Wu}$-Yang procedure will lead to conditions on quadruple overlaps.

The new feature of the two dimensional field theory is the existence of $\mathrm{Y}$ junctions when one subdivides the image of $\mathrm{S}$, see Fig. 3.4. The Wu-Yang prescription can be generalized in a simple manner to incorporate the physics of the $Y$ junctions. Assume that there are only two coordinate patches as in Fig. 3.5a. The Wu-Yang prescription is applicable. We now analyze what happens when one introduces a third patch $U_{\gamma}$ as in Fig. 3.5b. Note there are two Y junctions in the figure. The Wu-Yang prescription may be rewritten as:

$$
\int_{R_{\alpha}^{\prime}} T_{\alpha}-\int_{F_{1}} J_{\alpha \beta}-\int_{F_{2}} J_{\alpha \beta}+\int_{R_{\beta}^{\prime}} T_{\beta}-\int_{E_{\beta \gamma}} J_{\beta \gamma}+\int_{R_{\gamma}^{\prime}} T_{\gamma}-\int_{E_{\gamma \alpha}} J_{\gamma \alpha}-K_{\alpha \beta \gamma}\left(P_{1}\right)-K_{\alpha \beta \gamma}\left(P_{2}\right) \text {. }
$$

It is clear that the correct way to define the action in the case of $\mathrm{Y}$ junctions is

$$
\int_{\boldsymbol{R}_{\alpha}} T_{\alpha}-\int_{E_{\alpha \beta}} J_{\alpha \beta}+\int_{\boldsymbol{R}_{\beta}} T_{\beta}-\int_{E_{\beta \gamma}} J_{\beta \gamma}+\int_{R_{\gamma}} T_{\gamma}-\int_{E_{\gamma \alpha}} J_{\gamma \alpha}-K_{\alpha \beta \gamma}(P) .
$$

We have used the notation of Fig. 3.4. One can verify that a small movement of the $\mathrm{Y}$ junction such as in Fig. 3.6 leaves the value of the action invariant. This is the modification of the $\mathrm{Wu}$-Yang prescription that is required.

Let us now see what happens when one introduces a fourth patch, $U_{\delta}$, as in Fig. 3.7. Equation (3.13) may be rewritten as

$$
\begin{aligned}
\int_{R_{\alpha}^{\prime}} T_{\alpha} & +\int_{R^{\prime} \beta} T_{\beta}+\int_{R_{\gamma}^{\prime}} T_{\gamma}+\int_{R^{\prime} \delta} T_{\delta}-\int_{E_{\alpha \delta}} J_{\alpha \delta}-\int_{E_{\beta \delta}} J_{\beta \delta}-\int_{E_{\gamma \delta}} J_{\gamma \delta} \\
& -K_{\alpha \delta \gamma}\left(Q_{1}\right)-K_{\alpha \beta \delta}\left(Q_{2}\right)-K_{\beta \gamma \delta}\left(Q_{3}\right) \\
& -\left(K_{\alpha \beta \gamma}(P)+K_{\beta \gamma \delta}(P)+K_{\gamma \delta \alpha}(P)+K_{\delta \alpha \beta}(P)\right) .
\end{aligned}
$$



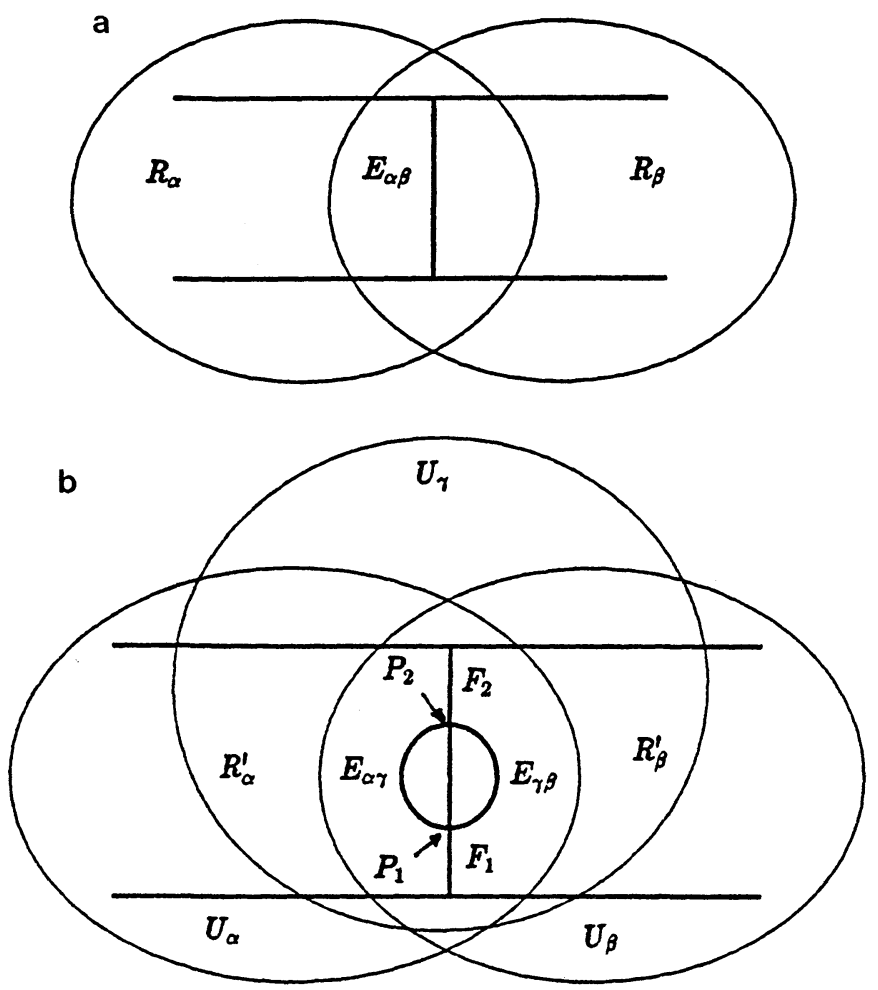

Fig. 3.5a. A schematic representation of Fig. 3.1. b Required subdivision of Fig. 3.5a when one introduces a third coordinate patch $U_{\gamma}$ (not depicted). The integration regions $R_{\alpha}$ and $R_{\beta}$ have been subdivided into three regions $R_{\alpha}^{\prime}, R_{\beta}^{\prime}$, and $R_{\gamma}^{\prime}$. The latter being the circular region with boundary $E_{\alpha \gamma}$ and $E_{\gamma \beta}$. The edge $E_{\alpha \beta}$ has been subdivided into $F_{2}, E_{\alpha \beta}^{\prime}$ and $F_{1}$ (going from top to bottom). The two Y-junctions are at the points $P_{1}$ and $P_{2}$ and do not include the edge $E_{\alpha \beta}^{\prime}$

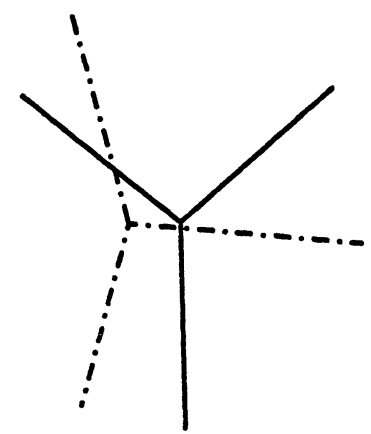

Fig. 3.6. A small movement of the Y-junction

The last line is a constant (independent of $P$ ). This may be derived by adding cyclic permutations of Eq. (3.11). We learn that just as in the electromagnetic case the classical action is defined up to an additive constant. A path integral quantization immediately tells us that a consistent quantum theory is only possible if

$$
K_{\alpha \beta \gamma}(P)+K_{\beta \gamma \delta}(P)+K_{\gamma \delta \alpha}(P)+K_{\delta \alpha \beta}(P)=2 \pi n_{\alpha \beta \gamma \delta},
$$




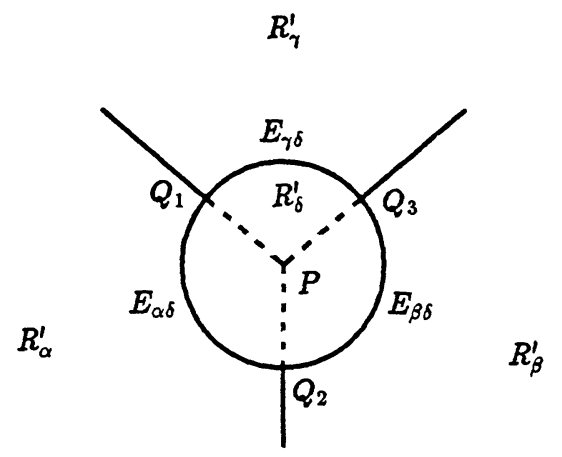

Fig. 3.7. Introduction of a fourth coordinate patch into Fig. 3.4. The $R$ regions have been sub divided into $R^{\prime}$ regions

where the $n$ 's are integers. Conditions have to be imposed on quadruple overlaps. Namely, one has to be able to choose a collection of integers $n_{\alpha \beta \gamma \delta}$ on any good cover of the manifold.

Finally, we mention that there is an analogue of the magnetic field in this problem. Consider the three forms $\left\{d T_{\alpha}\right\}$. This collection defines a global three form $\mathscr{G}$ as may be seen by noting that on an overlap $d T_{\alpha}=d T_{\beta}$. Therefore, one can define $\mathscr{G}$ as the differential of $T_{\alpha}$ on each coordinate patch. If one calculates the "flux" by integrating $\mathscr{G}$ over a boundaryless three dimensional region then one discovers that the total flux is given by the sum of $2 \pi n_{\alpha \beta \gamma \delta}$ over the patches that intersect the region of interest.

We have seen that the two dimensional field theory is very similar to the electromagnetic example except that certain modifications must take place. These ideas can be generalized to arbitrary dimensionality for spacetime. If spacetime is a $d$ dimensional manifold then one has to impose conditions on $(d+2)$-fold overlaps to understand the quantum theory.

It is possible to discuss the higher dimensional cases using the methods described in this section. This becomes extremely tedious. There is a very useful way of cataloguing all this information using a branch of mathematics called Cech cohomology. The next section introduces the ideas of Cech cohomology using these examples as a motivation.

\section{Cech Cohomology}

Cech cohomology is the correct language for formulating the examples presented in the two previous sections. The machinery of Cech cohomology provides a means of cataloguing the necessary information required to extract the physics. In this section we will explain the relationship of Cech cohomology to the topology of the manifold, and we will also explain the cataloguing procedure. We will not present Cech cohomology in its most abstract setting. The general theory will be stripped down to a level sufficient to attack and solve the problems addressed in this paper. We assume the reader is familiar with the elementary aspects of simplicial homology $[11,12]$. Namely, the concept of simplices, the existence of 


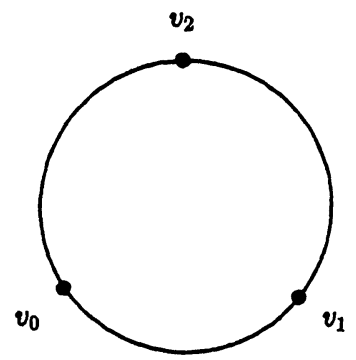

Fig. 4.1. Triangulation of a circle with vertices $v_{0}, v_{1}$, and $v_{2}$

triangulations of a manifold, the notion of a chain (the formal sum of simplices), and the concept of the boundary of a chain. A brief example is provided below.

For our purposes, simplicial homology is the study of the triangulations of a manifold. Simplices are generalizations of triangles. For example, a zero-simplex is a point, a one-simplex is a segment, a two-simplex is a triangle, a three-simplex is a tetrahedron, etc. The allowed triangulations of a manifold are determined by the topology of the manifold. Small deformations of the manifold will not change the triangulation. A triangulation of the circle is presented in Fig. 4.1. Note that there are three vertices $v_{0}, v_{1}$, and $v_{2}$, and three one-simplices $v_{1} v_{0}, v_{2} v_{1}$, and $v_{0} v_{2}$. There are no two simplices since the circle does not include its interior. A simplicial zero chain is a formal linear combination of vertices with integral coefficients. For example, consider the zero-chain $v_{0}+12 v_{1}-496 v_{2}$. Likewise a simplicial one chain is a linear combination of the one-simplices. For example, consider the onechain $2 v_{1} v_{0}+5 v_{2} v_{1}-13 v_{0} v_{1}$. The simplices are oriented. This is expressed by the definition that $v_{0} v_{1}=-v_{1} v_{0}$. The simplicial boundary of the one-simplex $v_{1} v_{0}$ is given by $\partial\left(v_{1} v_{0}\right)=v_{1}-v_{0}$, where $\partial$ denotes the boundary operator. A cycle is a chain $c$ with no boundary $\partial c=0$. A boundary $b$ is a chain which is the boundary of another chain $b=\partial c^{\prime}$. The boundary operation satisfies $\partial^{2}=0$.

It is clear that the topology of the manifold will determine the allowed triangulations and that there are many possible triangulations. What is remarkable is that there are certain invariants which are independent of the triangulations. These invariants are the homology groups. The homology groups are equivalence classes just like the homotopy groups. Two chains $c_{1}$ and $c_{2}$ are homologous if they differ by a boundary, $c_{1}-c_{2}=\partial c^{\prime}$ for some $c^{\prime}$. Geometrically this means that one can fill up the region between the chains as illustrated in the annulus example of Fig. 4.2. In other words, there are no holes in between the chains. Naively, the homology groups count holes in a manifold. They also count more complicated objects such as twistings as may be seen by studying the example of a projective space. Let $Z_{p}^{\Delta}$ be the set of all p-cycles in a triangulation of a manifold $M$. The triangle is used as a reminder that these are defined by triangulations. Let $B_{p}^{\Delta}$ be the set of all p-chains which are boundaries. Note that since $\partial^{2}=0$ one has $B_{p}^{\Delta} \subset Z_{p}^{\Delta}$. Both these objects are abelian groups under addition. The equivalence classes under homology are given by looking at the quotient $H_{p}^{\Delta}(M)=Z_{p}^{\Delta} / B_{p}^{\Delta} . H_{p}^{\Delta}(M)$ is called the $p$-th simplicial homology group of the manifold. It is a triangulation invariant and thus it is determined by the topology. 


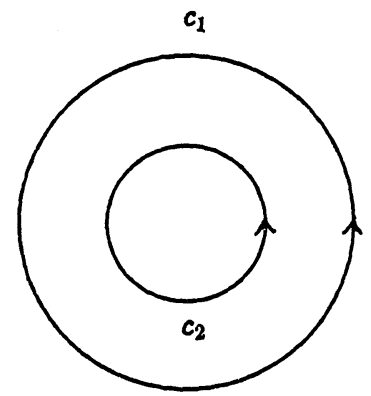

Fig. 4.2. Annular region bounded by chains $c_{1}$ and $c_{2}$

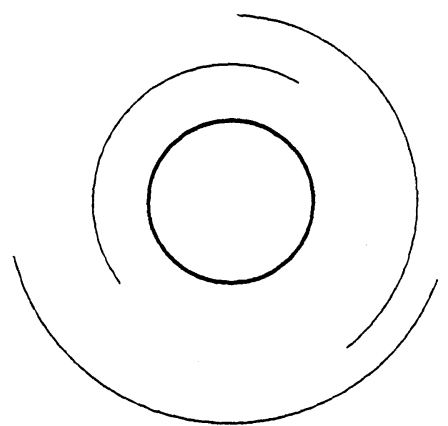

Fig. 4.3. A good cover for a circle. The three open sets are depicted externally for visualization purposes only

It is worthwhile to point out at this stage some differences between simplicial homology and homotopy. It can be shown that if $M$ is a manifold of dimension $n$, then $H_{p}^{\Delta}(M)$ vanish for $p>n$. In other words, there are no nontrivial chains of dimension exceeding the dimension of the manifold. This is not true in homotopy. For example, the Hopf fibration [7-13] which is responsible for ordinary monopoles shows that $\pi_{3}\left(S^{2}\right)=\mathbb{Z}$. Homotopy is not a statement about there being $a$ nontrivial three sphere in the two sphere. It is a statement about maps from the three sphere into the two sphere. Homology is the one that tells us that there are no nontrivial three dimensional chains in the two sphere. We will see that the topological charge quantization condition may be stated without reference to homotopy.

We will formulate Cech homology in a way that the connection to simplicial homology will be explicit. In all the manifolds we will consider it is always possible to choose an open cover $\mathscr{U}=\left\{U_{a}\right\}$ such that each open set and each non-empty finite intersection is diffeomorphic to an open ball in $\mathbb{R}^{n}[7]$. We will refer to these covers as good covers. At this stage we have already tailored Cech theory to the some specifics we require. Figure 4.3 illustrates such a cover for a circle. Note that a good cover for a circle requires at least three open sets. A major benefit of a good cover is that on each intersection the Poincaré lemma holds.

On each non-empty finite intersection define objects $U_{\alpha \beta}, U_{\alpha \beta \gamma}, U_{\alpha \beta \gamma \delta}$, etc. by

$$
\begin{aligned}
U_{\alpha \beta} & =U_{\alpha} \cap U_{\beta}, \\
U_{\alpha \beta \gamma} & =U_{\alpha} \cap U_{\beta} \cap U_{\gamma}, \\
U_{\alpha \beta \gamma \delta} & =U_{\alpha} \cap U_{\beta} \cap U_{\gamma} \cap U_{\delta} .
\end{aligned}
$$




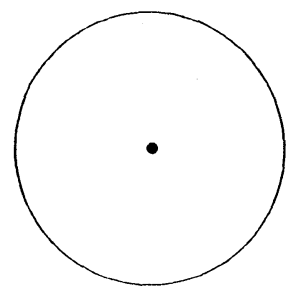

a

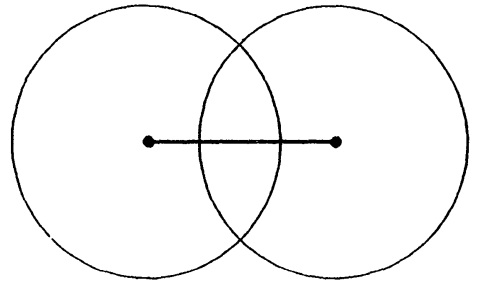

b

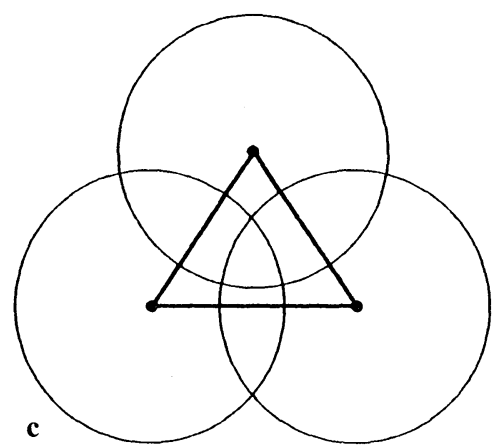

Fig. 4.4a. An open set and its associated vertex. b Two intersecting open sets and the associated segment. c Triply overlapping sets and the associated triangle

We define a formal orientation by requiring that $U_{\alpha \beta}=-U_{\beta \alpha}$, and likewise for the other objects. This good cover of the manifold defines a simplicial triangulation of the manifold. This is illustrated in Fig. 4.4. In each open set $U_{\alpha}$ we choose a point in the interior, see Fig. 4.4a. These points define the vertices of the triangulation. To each non-empty intersection we associate a one simplex, see Fig. $4.4 \mathrm{~b}$. To each non-empty triple intersection we associate a two simplex, see Fig. $4.4 \mathrm{c}$. It is clear that the combinatorics of a good cover defines a simplicial triangulation of the manifold.

By analogy one can define a Cech homology theory for the cover. The p-chains $C_{p}(\mathscr{U})$ are defined as formal linear combinations with integer coefficients of the "p-simplices" $\left\{U_{\alpha_{0} \alpha_{1} \cdots \alpha_{p}}\right\}$. The $\mathscr{U}$ is used to remind one that there is a dependence on the cover. One can define a boundary operation $\partial$ by $\partial U_{\alpha \beta}=U_{\alpha}-U_{\beta}$, and so forth for the higher chains. The p-cycles $Z_{p}(\mathscr{U})$ are the p-chains whose boundary is zero. The p-boundaries $B_{p}(\mathscr{U})$ are the $\mathrm{p}$-chains which are the boundary of a $(\mathrm{p}+1)$ chain. One can show that $\partial^{2}=0$. It follows that $B_{p}(\mathscr{U}) \subset Z_{p}(\mathscr{U})$. One can define the p-th homology group by $H_{p}(M)=Z_{p}(\mathscr{U}) / B_{p}(\mathscr{U})$. Since a good cover determines a triangulation, we must have that $H_{p}(M)=H_{p}^{\Delta}(M)$. The homology group is independent of the cover.

We motivated Cech homology by showing that it was related to simplicial homology. It is clear that the entire Cech homology machinery can be developed without ever referring to simplicial homology. It is not necessary to use a good cover. A good cover is the easiest way to see the relation to simplicial homology. Let us temporarily forget about the existence of simplicial homology. The allowed open covers for a manifold are determined by topological considerations. The Cech homology theory is determined by the combinatorial properties of the cover. Namely which cycles are not boundaries, etc. Therefore Cech homology contains topological information. It is a theorem that the Cech homology groups are independent of the cover ${ }^{1}$ one chooses [7]. There is a second theorem that asserts that the Cech homology groups are the same as the simplicial homology groups [7].

1 To obtain Cech homology groups which are independent of the cover one must take a direct limit, see [7]. If the cover is a good cover then the direct limit is not necessary 
Our main interest is not Cech homology theory but Cech cohomology theory. We will actually be dealing with cohomology with coefficients in a pre-sheaf but all this fancy language just obscures the main ideas. For our purposes, Cech cohomology provides a systematic way of cataloguing information and a systematic way of dealing with singular field configurations by avoiding the singularities. We will see how the ideas discussed in the two previous sections may be discussed in the language of Cech cohomology.

A p-chain with values in q-forms is an assignment of a nonsingular q-form to each p-chain. As an example consider the problem discussed in Sect. 2. In that case we assigned to each open set $U_{\alpha}$ a vector potential $A_{\alpha}$. The collection $\left\{A_{\alpha}\right\}$ is a zero cochain with values in one forms. We required that on $U_{\alpha}$ the vector potential $A_{\alpha}$ be nonsingular. $A_{\alpha}$ may be singular somewhere outside of $U_{\alpha}$. This singularity is the famous Dirac string singularity. The collection of gauge transformation $\left\{\psi_{\alpha \beta}\right\}$ defines a one cochain with values in the zero forms. In the two dimensional example of Sect. 3, we assigned to each open set a two form. Therefore the collection $\left\{T_{\alpha}\right\}$ defines a zero cochain with values in the two forms. Likewise, the collection of the transition functions $\left\{J_{\alpha \beta}\right\}$ defines a one cochain with values in the one forms.

We introduce some notational conventions. The $\mathrm{p}$-cochains with values in the q-forms will be denoted by $C^{p}\left(\mathscr{U}, \Omega^{q}\right)$. This notation explicitly shows the dependence on the good cover $\mathscr{U}$. The set of all $C^{p}\left(\mathscr{U}, \Omega^{q}\right)$ is related to the singular differential forms on the manifold. Note that the definition of $C^{p}\left(\mathscr{U}, \Omega^{q}\right)$ requires the forms to be nonsingular only on the associated chain. They can do whatever they want outside the chain. These objects are a subset of the singular differential forms on a manifold. It is clear from the examples of Sect. 2 and 3 that these are the objects which are of central importance to the question of topological charge quantization.

Let us try to answer the following question, "When does a zero cochain define a global differential form?" Consider a zero cochain $\left\{\lambda_{\alpha}\right\} \in C^{0}\left(\mathscr{U}, \Omega^{q}\right)$. The zero cochain specifies a q-form on each open set in the cover. Assume $U_{\alpha \beta} \neq 0$, then on the overlap $U_{\alpha \beta}$ one must have $\lambda_{\alpha}-\lambda_{\beta}=0$. If not then $\lambda_{\alpha}$ will not extend to $\lambda_{\beta}$. One can define a global differential q-form $\lambda^{\text {global }}$ if and only if $\left\{\lambda_{\alpha}-\lambda_{\beta}\right\}$ vanishes identically. Note that the collection $\left\{\lambda_{\alpha}-\lambda_{\beta}\right\}$ defines an element in $C^{1}\left(\mathscr{U}, \Omega^{q}\right)$. This already gives us an inkling on what Cech theory will do for us. It will in certain situations allow us to piece local information into global information. We will see that Cech cohomology provides a systematic way for determining when local information can be pieced into less local information.

The coboundary operator $\delta$ is an operation between $\mathrm{p}$-chains and $(p+1)$-chains:

$$
\delta: C^{p}\left(\mathscr{U}, \Omega^{q}\right) \rightarrow C^{p+1}\left(\mathscr{U}, \Omega^{q}\right) .
$$

It is defined as follows for small values of $\mathrm{p}$ :

$$
\begin{gathered}
\delta\left\{A_{\alpha}\right\}=\left\{A_{\alpha}-A_{\beta}\right\}, \\
\delta\left\{B_{\alpha \beta}\right\}=\left\{B_{\alpha \beta}+B_{\beta \gamma}+B_{\gamma \alpha}\right\}, \\
\delta\left\{C_{\alpha \beta \gamma}\right\}=\left\{C_{\alpha \beta \gamma}+C_{\beta \gamma \delta}+C_{\gamma \delta \alpha}+C_{\delta \alpha \beta}\right\} .
\end{gathered}
$$


The generalization to larger values of $\mathrm{p}$ is straightforward. One can show that the coboundary operator satisfies $\delta^{2}=0$. For completeness but not central to this exposition is the fact that the coboundary operator is the transpose of the boundary operator of Cech homology theory. This may be seen by noticing that a cochain is a linear operation from the space of chains into the space of local differential forms and therefore one can define a transpose to the boundary operation. The nilpotency of $\delta$ allows us to define a cohomology theory. We define the p-cocycles $Z_{\delta}^{p}\left(\mathscr{U}, \Omega^{q}\right)$ as those elements of $C^{p}\left(\mathscr{U}, \Omega^{q}\right)$ that are annihilated by $\delta$. A p-cocycle $z$ is said to be exact if one can find a $(\mathrm{p}-1)$-cochain $y$ such that $z=\delta y$. The p-coboundaries $B_{\delta}^{p}\left(\mathscr{U}, \Omega^{q}\right)$ are the image of $C^{p-1}\left(\mathscr{U}, \Omega^{q}\right)$ under $\delta$. One has that $B_{\delta}^{p}\left(\mathscr{U}, \Omega^{q}\right) \subset Z_{\delta}^{p}\left(\mathscr{U}, \Omega^{q}\right)$. This allows us to define cohomology classes $H_{\delta}^{p}\left(\mathscr{U}, \Omega^{q}\right)=Z_{\delta}^{p}\left(\mathscr{U}, \Omega^{q}\right) / B_{\delta}^{p}\left(\mathscr{U}, \Omega^{q}\right)$. Warning: these are not the objects that we will refer to as Cech cohomology classes! In fact we will see that these $H_{\delta}^{p}\left(\mathscr{U}, \Omega^{q}\right)$ are mostly trivial. This will play a central role in our discussion.

To see that the $H_{\delta}^{p}\left(\mathscr{U}, \Omega^{q}\right)$ are trivial it is necessary to introduce a partition of unity. A partition of unity is a collection of functions $\left\{p_{a}\right\}$, one function associated with each open set, satisfying the following properties:

1. $p_{\alpha} \geq 0$

2. $\Sigma_{\alpha} p_{\alpha}=1$

3. $p_{\alpha}$ has compact support in $U_{\alpha}$.

Assume that $\left\{J_{\alpha \beta}\right\}$ is in $Z_{\delta}^{p}\left(\mathscr{U}, \Omega^{q}\right)$, i.e., d $\left\{J_{\alpha \beta}\right\}=0$. It is a remarkable fact that every p-cocycle with $p>0$ is exact. To see how this comes about let us study our example with $\delta J=0$. The cocycle condition on $J$ states that

$$
\left\{J_{\alpha \beta}+J_{\beta \gamma}+J_{\gamma \alpha}\right\}=0 .
$$

Define a one cochain $K$ by

$$
K_{\alpha}=\sum_{\alpha} J_{\alpha \gamma} p_{\gamma} .
$$

Compute the coboundary of this cochain

$$
\begin{aligned}
\delta\left\{K_{\alpha}\right\} & =\left\{K_{\alpha}-K_{\beta}\right\}=\left\{\sum_{\gamma} J_{\alpha \gamma} p_{\gamma}-\sum_{\gamma} J_{\beta \gamma} p_{\gamma}\right\} \\
& =\left\{\sum_{\gamma}\left(J_{\alpha \gamma}+J_{\gamma \beta}\right) p_{\gamma}\right\}=\left\{\sum_{\gamma} J_{\alpha \beta} p_{\gamma}\right\}=\left\{J_{\alpha \beta}\right\} .
\end{aligned}
$$

In the above we used cocycle condition (4.6) and the antisymmetry in the indices. In fact we have explicitly demonstrated that $J$ is exact.

This construction generalizes to an arbitrary p-cocycle $\left\{J_{\alpha_{0} \alpha_{1} \ldots \alpha_{p}}\right\}$ with $p>0$. We can construct a (p-1)-chain $K$ such that $J=\delta K$. Define $K$ by

$$
\left\{K_{\alpha_{0} \alpha_{1} \ldots \alpha_{p-1}}\right\}=\left\{\sum_{\gamma} J_{\alpha_{0} \ldots \alpha_{p-1} \gamma} p_{\gamma}\right\} .
$$

It is a straightforward exercise to verify that $J=\delta K$. Since for $p>1$ we have that every p-cocycle is exact, the corresponding cohomology groups $H_{\delta}^{p}\left(\mathscr{U}, \Omega^{q}\right)$ must vanish. This theorem is similar to the Poincaré lemma for the exterior derivative. 
We will occasionally refer to it as the Poincare lemma for the $\delta$ operation. The proof of this lemma does not depend on $\mathscr{U}$ being a good cover.

The only case that remains is the case of $p=0$. We already discussed this situation. The zero cocycles are the global differential forms. There are no zero boundaries, therefore $H_{\delta}^{0}\left(\mathscr{U}, \Omega^{q}\right)=\Omega^{q}(M)$. One can use the following convenient device to provide a unified picture. Notice that a $\mathrm{p}$-cochain has $(\mathrm{p}+1)$ indices. A global differential form should contain zero indices since it is defined independent of the cover. The global differential forms $\Omega^{q}(M)$ may be identified with $C^{-1}\left(\mathscr{U}, \Omega^{q}\right)$. Given a zero cocycle one can use (4.9) to "write down" the global differential form. Notice that there is no $\delta$ operation from $C^{-1}\left(\mathscr{U}, \Omega^{q}\right)$ to $C^{0}\left(\mathscr{U}, \Omega^{q}\right)$. The corresponding operation is just the restriction of the global differential form to the open sets of the cover.

The machinery we have just presented is just a means of piecing together local information. For example, when can one piece together information on triple overlaps to construct quantities on the larger double overlaps? When one is given a two cocycle.

The final piece of formalism we need is the tic-tac-toe box [7]. We will be studying the so called double chain complexes. Note that the objects we have been discussing $C^{p}\left(\mathscr{U}, \Omega^{q}\right)$ are indexed by two integers. It is very convenient to arrange these in a table. We follow the conventions of Bott and Tu [7]. Unfortunately the index $\mathrm{p}$ is the column number and the index $\mathrm{q}$ is the row number. Note that there are two commuting operators that act on the entries in the box. Horizontally one has the coboundary operator $\delta$. Vertically one has the exterior derivative $d$. The leftmost column and the bottom row are used only for identification purposes.

\begin{tabular}{l|cccc}
$\Omega^{3}$ & $C^{0}\left(\mathscr{U}, \Omega^{3}\right)$ & $C^{1}\left(\mathscr{U}, \Omega^{3}\right)$ & $C^{2}\left(\mathscr{U}, \Omega^{3}\right)$ & $C^{3}\left(\mathscr{U}, \Omega^{3}\right)$ \\
$\Omega^{2}$ & $C^{0}\left(\mathscr{U}, \Omega^{2}\right)$ & $C^{1}\left(\mathscr{U}, \Omega^{2}\right)$ & $C^{2}\left(\mathscr{U}, \Omega^{2}\right)$ & $C^{3}\left(\mathscr{U}, \Omega^{2}\right)$ \\
$\Omega^{1}$ & $C^{0}\left(\mathscr{U}, \Omega^{1}\right)$ & $C^{1}\left(\mathscr{U}, \Omega^{1}\right)$ & $C^{2}\left(\mathscr{U}, \Omega^{1}\right)$ & $C^{3}\left(\mathscr{U}, \Omega^{1}\right)$ \\
$\Omega^{0}$ & $C^{0}\left(\mathscr{U}, \Omega^{0}\right)$ & $C^{1}\left(\mathscr{U}, \Omega^{0}\right)$ & $C^{2}\left(\mathscr{U}, \Omega^{0}\right)$ & $C^{3}\left(\mathscr{U}, \Omega^{0}\right)$ \\
\hline $\begin{array}{c}d \\
\delta\end{array}$ & $C^{0}$ & $C^{1}$ & $C^{2}$ & $C^{3}$ \\
$\delta$ & & & &
\end{tabular}

The abstract discussion of the tic-tac-toe boxes might not be very comprehensible at a first reading. It is suggested that the reader follow the examples that follow the abstract discussion. These examples are just the examples of Sects. 2 and 3 using the tic-tac-toe boxes as an accounting device.

The corresponding $\delta$-cohomology for each of the entries in the above box is given by the box directly below:

\begin{tabular}{ll|cccc}
$\Omega^{3}$ & & $\Omega^{3}(M)$ & 0 & 0 & 0 \\
$\Omega^{2}$ & & $\Omega^{2}(M)$ & 0 & 0 & 0 \\
$\Omega^{1}$ & & $\Omega^{1}(M)$ & 0 & 0 & 0 \\
$\Omega^{0}$ & & $\Omega^{0}(M)$ & 0 & 0 & 0 \\
\hline$d$ & $\uparrow$ & & & & \\
$\delta$ & $\rightarrow$ & $C^{0}$ & $C^{1}$ & $C^{2}$ & $C^{3}$
\end{tabular}


Let us look at the action of $d$ for a second:

$$
d: C^{p}\left(\mathscr{U}, \Omega^{q}\right) \rightarrow C^{p}\left(\mathscr{U}, \Omega^{q+1}\right) .
$$

Since $d^{2}=0$ one can define a cohomology theory with respect to $d$ acting on the $C^{p}\left(\mathscr{U}, \Omega^{q}\right)$. This operation moves us vertically. We can define the corresponding cohomology groups. The closed local differential forms $Z_{d}^{p}\left(\mathscr{U}, \Omega^{q}\right)$ are those elements of $C^{p}\left(\mathscr{U}, \Omega^{q}\right)$ that are annihilated by $d$. The exact forms $B_{d}^{p}\left(\mathscr{U}, \Omega^{q}\right)$ are the image of $C^{p}\left(\mathscr{U}, \Omega^{q-1}\right)$ under $d$. We can define cohomology groups by $H_{d}^{p}\left(\mathscr{U}, \Omega^{q}\right)=Z_{d}^{p}\left(\mathscr{U}, \Omega^{q}\right) / B_{d}^{p}\left(\mathscr{U}, \Omega^{q}\right)$. Most of these cohomology groups will vanish: $H_{d}^{p}\left(\mathscr{U}, \Omega^{q}\right)=0$ for $q=0$. Each non-empty multiple intersection of open sets is diffeomorphic to an open ball, and therefore the Poincare lemma is valid. Every local closed q-form, $q>0$, is locally exact. The exactness stops at the bottom row where the closed zero forms $Z_{d}^{p}\left(\mathscr{U}, \Omega^{0}\right)$ are given by locally constant functions according to the Poincaré lemma. Let us denote these objects by $C^{p}(\mathscr{U}, \mathbb{R})$. To be more explicit we note an element of $C^{p}(\mathscr{U}, \mathbb{R})$ is a collection $\left\{c_{\alpha_{0} \alpha_{1} \ldots \alpha_{p}}\right\}$, where each element in the collection is a real number. The $d$-cohomology of the tic-tac-toe box may be represented by the following diagram:

\begin{tabular}{|c|c|c|c|c|}
\hline$\Omega^{3}$ & 0 & 0 & 0 & 0 \\
\hline$\Omega^{2}$ & 0 & 0 & 0 & 0 \\
\hline$\Omega^{1}$ & 0 & 0 & 0 & 0 \\
\hline$\Omega^{0}$ & $\mathrm{C}^{0}(\mathscr{U}, \mathbb{R})$ & $C^{1}(\mathscr{U}, \mathbb{R})$ & $C^{2}(\mathscr{U}, \mathbb{R})$ & $C^{3}(\mathscr{U}, \mathbb{R})$ \\
\hline$d$ & & & & \\
\hline$\delta$ & $C^{0}$ & $C^{1}$ & $C^{2}$ & $C^{3}$ \\
\hline
\end{tabular}

For computational purposes it is convenient to write down an extended tic-tac-toe box. We will see the utility later. The external row and the external column next to the bars may be labelled by the index -1 .

\begin{tabular}{cc|cccc}
$\Omega^{3}$ & $\Omega^{3}(M)$ & $C^{0}\left(\mathscr{U}, \Omega^{3}\right)$ & $C^{1}\left(\mathscr{U}, \Omega^{3}\right)$ & $C^{2}\left(\mathscr{U}, \Omega^{3}\right)$ & $C^{3}\left(\mathscr{U}, \Omega^{3}\right)$ \\
$\Omega^{2}$ & $\Omega^{2}(M)$ & $C^{0}\left(\mathscr{U}, \Omega^{2}\right)$ & $C^{1}\left(\mathscr{U}, \Omega^{2}\right)$ & $C^{2}\left(\mathscr{U}, \Omega^{2}\right)$ & $C^{3}\left(\mathscr{U}, \Omega^{2}\right)$ \\
$\Omega^{1}$ & $\Omega^{1}(M)$ & $C^{0}\left(\mathscr{U}, \Omega^{1}\right)$ & $C^{1}\left(\mathscr{U}, \Omega^{1}\right)$ & $C^{2}\left(\mathscr{U}, \Omega^{1}\right)$ & $C^{3}\left(\mathscr{U}, \Omega^{1}\right)$ \\
$\Omega^{0}$ & $\Omega^{0}(M)$ & $C^{0}\left(\mathscr{U}, \Omega^{0}\right)$ & $C^{1}\left(\mathscr{U}, \Omega^{0}\right)$ & $C^{2}\left(\mathscr{U}, \Omega^{0}\right)$ & $C^{3}\left(\mathscr{U}, \Omega^{0}\right)$ \\
\hline$d$ & $\uparrow$ & $C^{0}(\mathscr{U}, \mathbb{R})$ & $C^{1}(\mathscr{U}, \mathbb{R})$ & $C^{2}(\mathscr{U}, \mathbb{R})$ & $C^{3}(\mathscr{U}, \mathbb{R})$ \\
$\delta$ & $\rightarrow$ & $C^{0}$ & $C^{1}$ & $C^{2}$ & $C^{3}$
\end{tabular}

The corresponding Poincare lemmas may not be used on the external entries. As far as the external column is concerned not every closed global differential form is exact. This means that the external column has a non-trivial $d$-cohomology. This cohomology is the standard cohomology of differential forms which is called 
DeRham cohomology and it will be denoted by $H_{D R}^{q}(M)$. The situation is more subtle on the external row. The objects of the bottom row are locally constant cochains. If $c \in C^{p}(\mathscr{U}, \mathbb{R})$ is a locally constant cocycle, then applying the prescription of the $\delta$ Poincaré lemma would lead to a cochain which was not locally constant. Remember that one has to multiply the cocycle by the corresponding partition of unity element. Since the $p_{\alpha}$ are not constant, the constructed element will not be locally constant and thus is not in $C^{p-1}(\mathscr{U}, \mathbb{R})$. The conclusion is that the external row has a nontrivial $\delta$-cohomology just like the external column. The cohomology of the external row is called the Cech cohomology of the manifold (with real coefficients), and it will be denoted by $H_{C}^{p}(M, \mathbb{R})$. There is a theorem [7] that states that the Cech cohomology classes are independent of the cover and only depend on the manifold. ${ }^{2}$ We will eventually show that the DeRham cohomology and the Cech cohomology are isomorphic. Before addressing this important question we turn to a demonstration of the utility of the tic-tac-toe boxes.

Consider the example of the magnetic monopole. It will not be necessary to assume that the electrically charged particle moves on the surface of a sphere. The configuration space for the trajectory of the particle may be any compact manifold without boundary. In the box below we have included the vector potentials and the transition functions,

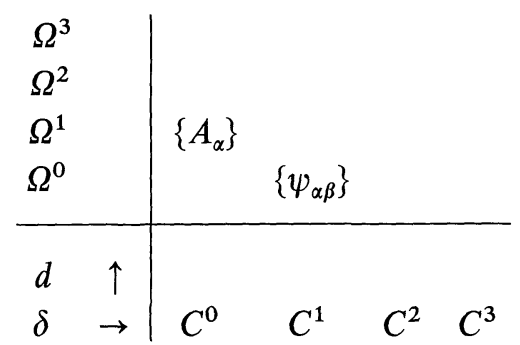

Let us apply the $d$ and the $\delta$ operations to the elements in the above box. We can operate again with $d$ and $\delta$ and get zero since these operators are nilpotent. Notice that one of the entries is the gauge transformation law.

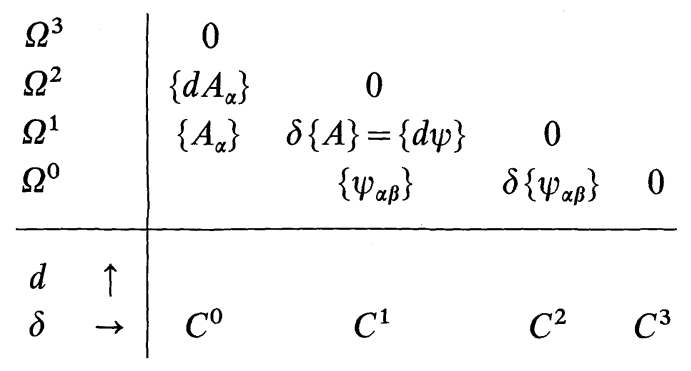

2 A precise statement of the theorem requires the notion of a direct limit, see [7] 
Define quantities $F_{\alpha}$ by $F_{\alpha}=d A_{\alpha}$, and $c_{\alpha \beta \gamma}$ by $\delta\left\{\psi_{\alpha \beta}\right\}=\left\{c_{\alpha \beta \gamma}\right\}$. The above box thus becomes:

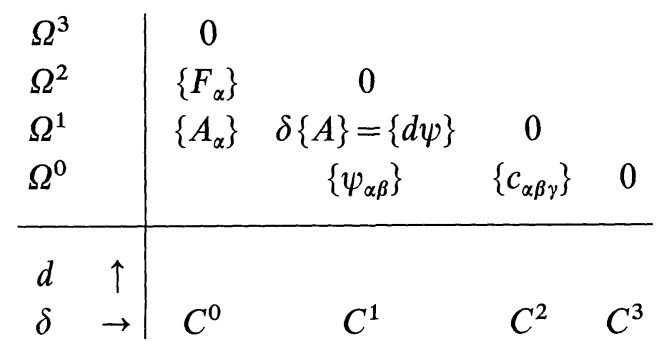

We immediately learn that the $F_{\alpha}$ is $d$-closed and it is also a zero $\delta$-cocycle. The means that $F_{\alpha}$ defines a closed global differential form $F$, the electro-magnetic field strength two form.

The other piece of information we learn from the tic-tac-toe box involves the $\left\{c_{\alpha \beta \gamma}\right\}$. These objects are $d$-closed and they define a two cocycle. Since locally closed zero form is given by a constant, the $\left\{c_{\alpha \beta \gamma}\right\}$ must define a two cocycle in $C^{2}(\mathscr{U}, \mathbb{R})$. All this information is shown in the box below.

\begin{tabular}{cc|cccc}
$\Omega^{3}$ & 0 & 0 & & & \\
$\Omega^{2}$ & $F$ & $\left\{F_{\alpha}\right\}$ & 0 & & \\
$\Omega^{1}$ & & $\left\{A_{\alpha}\right\}$ & $\delta\{A\}=\{d \psi\}$ & 0 & \\
$\Omega^{0}$ & & & $\left\{\psi_{\alpha \beta}\right\}$ & $\left\{c_{\alpha \beta \gamma}\right\}$ & 0 \\
\hline$d$ & $\uparrow$ & & & $\left\{c_{\alpha \beta \gamma}\right\}$ & 0 \\
$\delta$ & $\rightarrow$ & $C^{0}$ & $C^{1}$ & $C^{2}$ & $C^{3}$
\end{tabular}

The main conclusion is that given a collection of vector potentials $\left\{A_{\alpha}\right\}$ and transition functions $\left\{\psi_{\alpha \beta}\right\}$, the gauge transformation law $\delta A=\psi$, one can construct a closed global two form $F$ and a locally constant two cocycle $c$. $F$ is a representative of the second DeRham cohomology class. The two cocycle $c$ is a representative of the second Cech cohomology class of the manifold. Later we will see that there is an isomorphism between the DeRham classes and the Cech classes. Remember that the total magnetic flux through the manifold was determined by the $\left\{c_{\alpha \beta \gamma}\right\}$. There several notes of interest. The total magnetic flux through the sphere was determined by conditions on triple overlaps. Quantum mechanics imposes a further condition on the cocycle $\left\{c_{\alpha \beta \gamma}\right\}$. The $c$ 's must be $2 \pi \times$ (integer). This imposes a severe restriction on the cohomology classes. The integers $\mathbb{Z}$ are a subset of the real numbers $\mathbb{R}$. One can define objects $\left\{n_{\alpha \beta \gamma}\right\}$ to be integer valued cochains instead of real valued cochains. The set of the integer valued p-cochains will be denoted by $C^{p}(\mathscr{U}, \mathbb{Z})$. It is clear that $C^{p}(\mathscr{U}, \mathbb{Z})$ is a subset of $C^{p}(\mathscr{U}, \mathbb{R})$. Since the $\delta$ Poincaré lemma does not apply to real valued cochains then it certainly does not apply to the integer valued cochains. Therefore, there will be non-trivial cohomology in $C^{p}(\mathscr{U}, \mathbb{Z})$. These cohomology classes are called Cech cohomology classes with integer coefficients and they will be denoted by $H_{C}^{p}(M, \mathbb{Z})$. Quantum mechanics requires that the cocycle $\left\{c_{\alpha \beta \gamma} /(2 \pi)\right\}$ must be integral. The existence of 
such a cocycle is determined by whether or not the manifold in question admits integral cocycles in its second Cech cohomology class, i.e., $\mathbb{Z} \subset H_{C}^{2}(M, \mathbb{Z})$. The existence of such integral cocycles is determined by the topology of the manifold. The magnetic flux will be quantized if the manifold admits a second cohomology class with integer coefficients that contains the integers.

The situation becomes more interesting when one looks at the two dimensional example. In the tic-tac-toe box below we have included the Lagrangian and its gauge transformation properties.

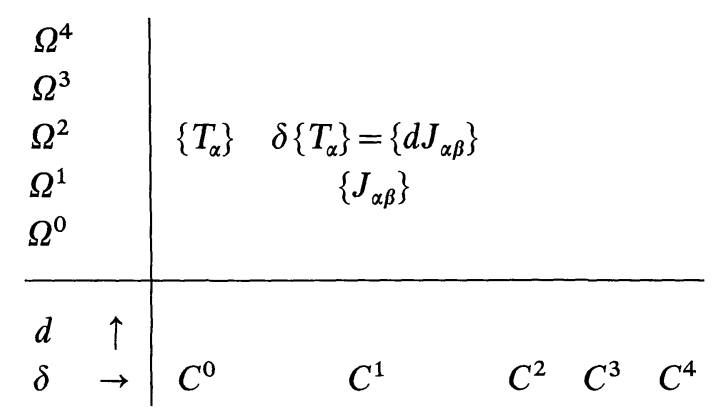

First we record the consequence of multiple $d$ and $\delta$ operations. This is show the box below:

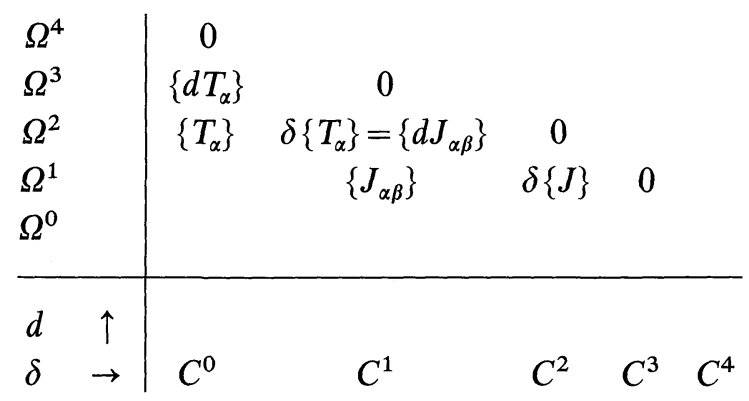

One of the pieces of information we have is that $\delta J$ is closed, $d \delta J=0$. This follows from the commutativity of the two operations. The tic-tac-toe box automatically takes this into account. Since $\delta J$ is closed and since the cohomology is trivial, there must exist a $K \in C^{2}\left(\mathscr{U}, \Omega^{0}\right)$ such that $J$ is its differential. This is illustrated in the box below

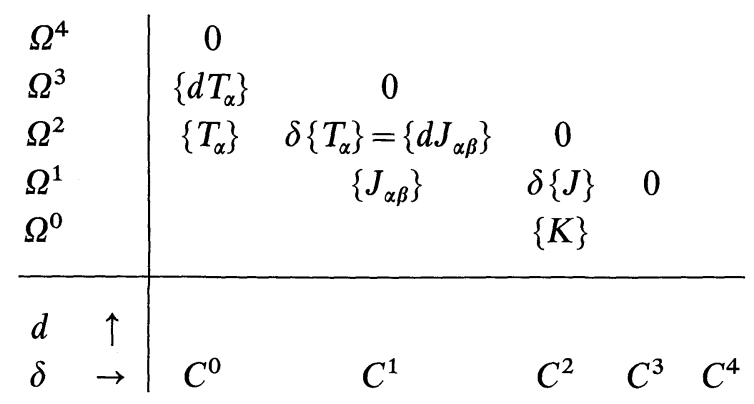


Applying the $d$ and $\delta$ information to the box above we find:

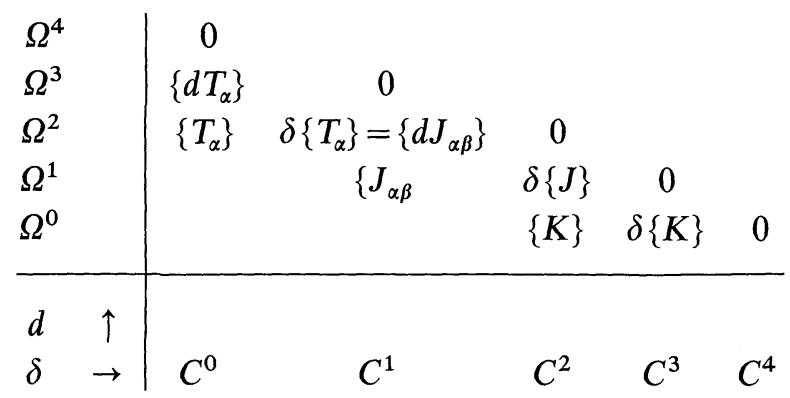

We learn that $\delta K$ is a closed three-cocycle. This cocycle is represented by constant cocycle $\left\{c_{\alpha \beta \gamma \delta}\right\}=\delta K$. The other piece of information we need to know is that $\left\{d T_{\alpha}\right\}$ defines a closed global differential form $\mathscr{G}$. This is all depicted in the box below.

\begin{tabular}{cc|ccccc}
$\Omega^{4}$ & 0 & 0 & & & & \\
$\Omega^{3}$ & $\mathscr{G}$ & $\left\{d T_{\alpha}\right\}$ & 0 & & & \\
$\Omega^{2}$ & & $\left\{T_{\alpha}\right\}$ & $\delta\left\{T_{\alpha}\right\}=\left\{d J_{\alpha \beta}\right\}$ & 0 & & \\
$\Omega^{1}$ & & & $\left\{J_{\alpha \beta}\right\}$ & $\delta\{J\}$ & 0 & \\
$\Omega^{0}$ & & & & $\{K\}$ & $\delta\{K\}$ & 0 \\
\hline$d$ & $\uparrow$ & & & & $\left\{c_{\alpha \beta \gamma \delta}\right\}$ & 0 \\
$\delta$ & $\rightarrow$ & $C^{0}$ & $C^{1}$ & $C^{2}$ & $C^{3}$ & $C^{4}$
\end{tabular}

Just as in the electromagnetic case, the Lagrangian and its gauge transformation law determines a closed global differential form and a locally constant cocycle. It is also clear from previous discussions that the integral of $\mathscr{G}$ over a boundaryless three dimensional region will be given by the sum of the $c_{\alpha \beta \gamma \delta}$ that belong to a covering of the region. A consistent quantum theory requires that the cochain be integral. The quantum mechanical theory can only be defined on a manifold whose third Cech cohomology class with integer coefficients contains the integers.

We have seen that the tic-tac-toe boxes and the ideas of Cech cohomology are the correct way to phrase the ideas of Sect. 2 and 3. It is clear that these ideas generalize to higher dimensional situations. We now turn to some more mathematics and in Sect. 5 we return to physics.

We will now illustrate how one shows the equivalence of DeRham cohomology and Cech cohomology. We work out the simplest non-trivial case since all the more complicated cases use the same techniques. Assume one is interested in the second DeRham cohomology class of the manifold. Let $F$ be a representative of this class. Note that $F$ is closed. This is shown below. 


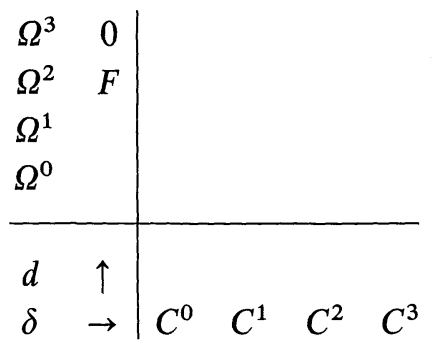

We can move $F$ to the inside of the box by defining $F_{\alpha}$ to be the restriction of $F$ to $U_{\alpha}$. Notice that the global nature of $F$ is explicit.

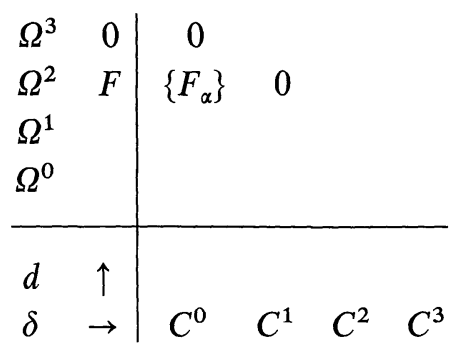

Next we use the fact that the Poincaré lemma can be used inside the box to define objects $A_{\alpha}$.

\begin{tabular}{ll|cccc}
$\Omega^{3}$ & 0 & 0 & & & \\
$\Omega^{2}$ & $F$ & $\left\{F_{\alpha}\right\}$ & 0 & & \\
$\Omega^{1}$ & & $\left\{A_{\alpha}\right\}$ & & & \\
$\Omega^{0}$ & & & & & \\
\hline$d$ & $\uparrow$ & & & & \\
$\delta$ & $\rightarrow$ & $C^{0}$ & $C^{1}$ & $C^{2}$ & $C^{3}$
\end{tabular}

We apply $\delta$ twice to obtain:

\begin{tabular}{lc|cccc}
$\Omega^{3}$ & 0 & 0 & & & \\
$\Omega^{2}$ & $F$ & $\left\{F_{\alpha}\right\}$ & 0 & & \\
$\Omega^{1}$ & & $\left\{A_{\alpha}\right\}$ & $\delta\{A\}$ & 0 & \\
$\Omega^{0}$ & & & & & \\
\hline$d$ & $\uparrow$ & & & & \\
$\delta$ & $\rightarrow$ & $C^{0}$ & $C^{1}$ & $C^{2}$ & $C^{3}$
\end{tabular}


We can use the exactness of $d$ inside the box to find objects $\psi_{\alpha \beta}$ such that $\left\{d \psi_{\alpha \beta}\right\}=\delta\left\{A_{\alpha}\right\}$. This is shown below.

\begin{tabular}{cc|cccc}
$\Omega^{3}$ & 0 & 0 & & & \\
$\Omega^{2}$ & $F$ & $\left\{F_{\alpha}\right\}$ & 0 & & \\
$\Omega^{1}$ & & $\left\{A_{\alpha}\right\}$ & $\delta\{A\}$ & 0 & \\
$\Omega^{0}$ & & & $\left\{\psi_{\alpha \beta}\right\}$ & & \\
\hline$d$ & $\uparrow$ & & & & \\
$\delta$ & $\rightarrow$ & $C^{0}$ & $C^{1}$ & $C^{2}$ & $C^{3}$
\end{tabular}

Applying $\delta$ twice we find:

\begin{tabular}{cc|cccc}
$\Omega^{3}$ & 0 & 0 & & & \\
$\Omega^{2}$ & $F$ & $\left\{F_{\alpha}\right\}$ & 0 & & \\
$\Omega^{1}$ & & $\left\{A_{\alpha}\right\}$ & $\delta\{A\}$ & 0 & \\
$\Omega^{0}$ & & & $\left\{\psi_{\alpha \beta}\right\}$ & $\delta\{\psi\}$ & 0 \\
\hline$d$ & $\uparrow$ & & & & \\
$\delta$ & $\rightarrow$ & $C^{0}$ & $C^{1}$ & $C^{2}$ & $C^{3}$
\end{tabular}

We conclude that $\delta \psi$ is a constant two cocycle $\left\{c_{\alpha \beta \gamma}\right\}$ in $C^{2}(\mathscr{U}, \mathbb{R})$. The two cocycle defines an element in $H_{C}^{2}(M, \mathbb{R})$. Summarizing, we have shown that given an element in the DeR ham cohomology class one can construct a element in the Cech class by zig-zagging through the box.

One can use the same type of tricks to show that given an element of the Cech cohomology class one can construct an element of the DeRham class. The only thing that remains is to show the one to one nature. For example, this requires showing that something which is zero in DeRham cohomology leads to something which is zero in Cech cohomology. The zero element in DeRham cohomology may be represented by an exact global differential form. One now uses this differential form to start a zig-zag pattern through the box and deduce the existence of a $b$ such that $c=\delta b$. One can also prove the converse in a similar fashion.

In conclusion, we have illustrated how one proves the isomorphism between DeRham cohomology and Cech cohomology. We emphasize that every DeRham cohomology class can be represented as a Cech cohomology class, and every Cech cohomology class can be represented as a DeRham cohomology class.

There is something that one learns by looking at the zig-zag patterns used in proving the equivalence of the cohomology theories. The cohomology representative determines the local Lagrangians $\left\{T_{\alpha}\right\}$, the gauge functions $\left\{J_{\alpha \beta}\right\}$ and the transformation law $\delta\{T\}=\{d J\}$. We had previously shown the converse in our discussion of the examples of Sects. 2 and 3.

We finish this section with a discussion of the relationship of Cech cohomology with integer coefficients to Cech cohomology (with real coefficients). The relationship is given by something called the universal coefficient theorem $[7 ; 14]$. 
We will not prove this theorem but we will apply it to the case at hand. Remember that if $V$ and $W$ are vector spaces then one can define the tensor product $V \otimes W$. If $s$ is a scalar then in an obvious notation one has $(s v) \otimes w=v \otimes(s w)=s(v \otimes w)$. For our purposes, the following imprecise form ${ }^{3}$ of the universal coefficient theorem illustrates what we have to know:

$$
H_{C}^{p}(M, \mathbb{Z}) \otimes \mathbb{R}=H_{C}^{p}(M, \mathbb{R}) .
$$

As we will see, the integer cohomology classes contain more information than the real cohomology classes. This can be seen by making use of two pieces of information. Firstly, one has the following important lemma:

$$
\mathbb{Z}_{n} \otimes \mathbb{R}=0 \text {. }
$$

To prove this one notices that if $r \neq 0$ and if $k \in \mathbb{Z}_{n}$ then $k \otimes r=k \otimes n(r / n)$ $=n(k \otimes(r / n))=(n k) \otimes(r / n) \equiv 0 \otimes(r / n) \equiv 0$. Secondly, one needs a theorem [15] that states that the integer classes are of the form

$$
H_{C}^{p}(M, \mathbb{Z})=\mathbb{Z} \oplus \cdots \oplus \mathbb{Z} \oplus \mathbb{Z}_{n_{1}} \oplus \cdots \oplus \mathbb{Z}_{n_{j}} .
$$

The $\mathbb{Z}_{n}$ pieces are called torsion pieces or finite pieces. When one tensors with the reals one loses all the torsion pieces according to the lemma. Therefore, the real cohomology classes are obtained from the integer cohomology classes by throwing out all torsion pieces and replacing the integers by the reals.

As a bonus to our entire discussion, a torsionless element of $H_{C}^{p}(M, \mathbb{Z})$ can be represented as a differential form. The integral of such a differential form over any boundaryless region will always give an integer. Torsion elements cannot be represented as differential forms.

\section{The Physics in Cech Cohomology}

From the viewpoint of a physicist, the fundamental object in a dynamical theory is the Lagrangian. The ideas discussed in this paper are descendants of the familiar theorem in classical mechanics on the non-uniqueness of the Lagrangian by the inclusion of total time derivatives. Spacetime will be a manifold $S$ of dimension $d$ with no "spatial" boundary. Possible examples are $\mathbb{R} \times S^{d-1}$ or $S^{d}$ or $\mathbb{R} \times T^{d-1}$, etc. The fields will live in a manifold $M$ of dimension $n$ which we assume is compact and without boundary.

We are interested in theories whose Lagrangian may be split into two parts

$$
L=L_{0}+T \text {. }
$$

The first term, $L_{0}$, is a well defined Lagrangian by itself. It can be a kinetic energy term with interactions. It is worthwhile noting that $L_{0}$ is typically a density and not a differential form. In other words, under a change of variables in spacetime, $L_{0}$ changes by the absolute value of the Jacobian and not the Jacobian itself.

The second term $T$ will be referred to as the topological term or the topological Lagrangian. It has several distinct features that lead to new properties of the

3 Technically, $H_{C}^{p}(M, \mathbb{Z})$ is a module and not a vector space 
theory. It is actually a differential form and thus it changes by the Jacobian of the spacetime coordinate transformation. More importantly, it must be interpretable as the pull back to $S$ of a differential form on $M$. The interesting properties arise when one does not require $T$ to be globally defined. One exploits the nonuniqueness theorem of the Lagrangian by considering a family of topological Lagrangians $\left\{T_{\alpha}\right\}$. One $T_{\alpha}$ for each open set is a cover for the manifold. The local topological Lagrangians are required to agree on overlaps up to a total derivative. This means that on each non-empty overlap $U_{\alpha \beta}$ one gives a transition form $J_{\alpha \beta}$ such that

$$
T_{\alpha}-T_{\beta}=d J_{\alpha \beta}
$$

in the overlap. In the language of Cech cohomology, the topological term is a cochain in $C^{0}\left(\mathscr{U}, \Omega^{d}\right)$, where $d$ is the dimension of spacetime. The collection of transition functions is an element of $C^{1}\left(\mathscr{U}, \Omega^{d-1}\right)$. These objects are related by the condition that

$$
\delta\left\{T_{\alpha}\right\}=\left\{d J_{\alpha \beta}\right\} .
$$

The objects thus defined depend on the choice of a good cover.

It is possible to generalize the $\mathrm{Wu}-\mathrm{Yang}$ construction to the $d$ dimensional case. One has to consider the generalizations of Y junctions and appropriate terms must be introduced into the action. The appropriate terms can all be picked up from the tic-tac-toe box. One starts zig-zagging to the right and down. One will need all terms of the form $C^{p}\left(\mathscr{U}, \Omega^{d-p}\right)$, where $0 \leq p \leq d$ to construct the generalized WuYang action. As in the examples we discussed in detail one finds that there is an ambiguity in the classical action when one looks at what happens in $(d+2)$-fold multiple overlaps. This ambiguity is given by a locally constant cocycle $\{c\}$ in $C^{d+1}\left(\mathscr{U}, \Omega^{0}\right)$. This cocycle defines a cohomology class in $H_{C}^{d+1}(M, \mathbb{R})$. There will be a Wu-Yang ambiguity in the action if $H_{C}^{d+1}(M, \mathbb{R})$ contains the reals.

Quantum mechanics imposes a constraint on the theory. The cocycle in $C^{d+1}\left(\mathscr{U}, \Omega^{0}\right)$ must be $2 \pi$ times integers in order to have a well defined path integral. This means that $H_{C}^{d+1}(M, \mathbb{Z})$ must contain the integers.

If we zig-zag up and to the left beginning from the $T$ 's then we can construct a closed global differential form $\mathscr{G}$ which defines an element in $H_{D R}^{d+1}(M)$. Since the Cech and the DeRham cohomology theories are isomorphic, the topological information contained in $\{c\}$ is the same as the one contained in $\mathscr{G}$. The total " $\mathscr{G}$ flux" through a boundaryless $d+1$ dimensional submanifold is given by the sum of the $c$ 's that belong to a cover of the submanifold. In the quantum theory, these $c$ 's have to be $2 \pi$ times integers and therefore we conclude that the total flux must be $2 \pi$ times an integer.

The universal coefficient theorem leads to an insight on the relationship of the classical theory to the quantum theory. The real Cech cohomology group $H_{C}^{d+1}(M, \mathbb{R})$ can be constructed if one knows the integer cohomology group $H_{C}^{d+1}(M, \mathbb{Z})$. If the integer cohomology group is pure torsion then the real cohomology group vanishes. This means that there is no ambiguity in the classical action. More precisely, an ambiguity at the $d+2$ overlap level will be the $\delta$ of something and can thus be absorbed into redefinition of what happens at the $d+1$ overlap level. The quantum theory in this case is also very dull since the flux 
through any $d+1$ dimensional closed surface will be zero. If the integer cohomology group $H_{C}^{d+1}(M, \mathbb{Z})$ contains the integers, then the real cohomology group $H_{C}^{d+1}(M, \mathbb{R})$ will contain the reals. If there is an ambiguity in the definition of the action in the classical theory then there will automatically be a flux quantization condition in the quantum theory.

The ambiguity in the classical action can be regarded as a cocycle representing an element of $H_{C}^{d+1}(M, \mathbb{R})$. When $H_{C}^{d+1}(M, \mathbb{R}) \neq 0$, this ambiguity is interesting. Quantum mechanics requires that the cocycle also represents an element in $H_{C}^{d+1}(M, \mathbb{Z})$. This requirement restricts the allowed class of Lagrangians due to the quantization of the associated flux. ${ }^{4}$

The formulation of the physics in terms of the local Lagrangians is a bit unsatisfactory since one has to choose a cover of the manifold and the Lagrangians in some sense are determined by the cover. It is more aesthetically satisfactory to formulate the theory by stating that the fundamental concept is the existence of a non-trivial class in $H_{D R}^{d+1}(M)$. Such a class is represented by a global closed differential form $\mathscr{G}$. Given a good cover $\mathscr{U}, \mathscr{G}$ may be locally represented by forms $T_{\alpha}$ such that $\mathscr{G}=d T_{\alpha}$. We are now back at the Lagrangian level.

In the previous section we showed that three viewpoints are equivalent. One could begin with an element of $H_{D R}^{d+1}(M)$ and by the use of the tic-tac-toe box construct the Lagrangian, the transition functions, the additional information required in the $\mathrm{Wu}-\mathrm{Yang}$ procedure, and the Cech cohomology class. Equivalently one could begin with the Cech cohomology class and construct all the above by using the tic-tac-toe box. Finally, one could take the local Lagrangians, the transition functions, the transformation law and with the aid of the tic-tac-toe box construct the remaining objects and the cohomology classes. The choice of a starting point is a personal preference.

The cohomological viewpoint leads to quantization conditions for many theories. The Wess-Zumino topological Lagrangian [3] is a special case of the general theory. Wess-Zumino type Lagrangians play a special role since they are derived by using group theoretical considerations and are usually associated with some homogeneous space $G / H$, where $G$ and $H$ are Lie groups. If the $(d+1)$ cohomology group of the homogeneous space contains the integers then there will be a Wess-Zumino term with quantized coupling. The strength of the cohomological theory presented in this paper lies in the fact that topological Lagrangians exist even in manifolds that have no obvious connection to group theory. Note that if the dimension of $M$ is one more than the dimension of spacetime $S$ then there will automatically be a topological quantization condition since $H_{D R}^{d+1}(M)=\mathbb{R}$ by Poincaré duality [7].

The famous three dimensional mass term for Yang-Mills theories [5] has a quantized coupling constant [4]. The quantization condition can be derived by using Cech cohomology. The situation in this case is analogous to the magnetic monopole discussion of Sect.2. Spacetime $S$ is a suitable three dimensional manifold. Let $M$ be a compact manifold of dimension greater than or equal to four. Assume there is a Yang-Mills connection defined on it. We will view $S$ as being embedded in $M$. The four form $\mathscr{G}=F \wedge F$ represents an element in the fourth

4 I would like to thank the referee for suggesting this clear paragraph 
DeRham cohomology class of the manifold. If this class is nontrivial then the associated three dimensional local Lagrangians will have a quantized coupling constant.

In fact, it is even possible to get a quantized mass term in the abelian case if the topology is complicated enough. Assume $S$ has the topology of $S^{2} \times S^{1}$, and assume that $M$ is $S^{2} \times S^{2}$ with a magnetic monopole in each of the $S^{2}$ factors. This manifold has a nontrivial fourth cohomology class which can be represented by the four form which is the wedge product of the respective electromagnetic field two forms on each of the two spheres.

The higher dimensional analog of the above is easily constructed. Assume $S$ is an odd dimensional manifold and $M$ is a manifold of dimension greater than equal to $d+1$. Assume there is a Yang-Mills connection on $M$. If we think of $S$ as being embedded in $M$ then it is easy to construct the global differential form that leads to the local Lagrangian. Consider the $(d+1) / 2$-fold wedge product

$$
\mathscr{G}=F \wedge \cdots \wedge F .
$$

This represents an element of the $d+1$ cohomology class of the manifold. If this class is nontrivial then the associated $d$ dimensional Lagrangian will have a quantized coefficient. Note that the example of Sect. 2 is just the $d=1$ case of the above discussion, and the three dimensional Yang-Mills mass term is the $d=3$ case. In eleven dimensions one would have an interaction which includes terms of the type $A F^{5}$ where the wedge product is understood.

One can also get a charge quantization in antisymmetric tensor field theories. Assume spacetime $S$ is a manifold of dimension $d=2 k+1$. Let $\mathscr{A}$ be a $k$-form and let $\mathscr{F}=d \mathscr{A}$. This antisymmetric tensor field theory has a mass term given by $\mathscr{A} \wedge \mathscr{F}$. This theory has an abelian gauge invariance and the mass term changes by a total derivative under this gauge invariance. By complicating the topology enough this term can be quantized. Here is one of many possible examples. Assume $d=4 j-1$ then $\mathscr{A}$ is a $(2 j-1)$ form and $\mathscr{F}$ is a $2 j$ form. Assume $S$ is given by $S^{2} \times S^{2} \times \cdots \times S^{2} \times S^{1}$ and $M$ is the $2 j$-fold Cartesian product of two spheres. Assume there is a magnetic monopole in each of the two spheres in $M$. Let $F$ be the total electromagnetic field two form. Define $\mathscr{F}$ by $\mathscr{F}=F^{j}$. This object is closed and thus represents a cohomology class. The $4 j$ form we need for charge quantization is just $\mathscr{F}^{2}$. This $4 j$ cohomology class is nontrivial.

The ideas of Rabinovici et al. [16] can also be seen from the Cech cohomological viewpoint.

The Bagger-Witten quantization condition [6] for the gravitational constant in certain supergravity theories can also be phrased in the language of Cech cohomology. In this situation one can draw tic-tac-toe boxes for the Kahler potential. We will not discuss this example in detail.

\section{Cohomology versus Homotopy}

In this section we show that cohomology is the more important concept in the question of topological quantization. We will construct examples where the homotopy criteria fails but where there is a charge quantization. We first review 
Witten's quantization argument [2]. In his seminal paper on the Wess-Zumino Lagrangian, Witten described how homotopy could be used to argue for coupling constant quantization. Witten assumes that spacetime $S$ is a $d$-sphere $S^{d}$. The sphere is mapped into the manifold $M$ by the field $\phi$. If $\pi_{d}(M)$ vanishes then it is possible to extend the map $\phi$ to the interior of a $d+1$ dimensional disk $D^{d+1}$ whose boundary is the image of spacetime. If $\pi_{d+1}(M)$ contains the integers then there must exist a generating sphere for the homotopy group. There will be an obstruction to large motions of the disk and therefore an ambiguity to how one extends the map $\phi$. Since there is a homotopically non-trivial $S^{d+1}$ then by DeRham's theorem there must exist a closed differential form $\omega$ such that

$$
\int_{S^{d+1}} \omega=1 \text {. }
$$

Witten argues that a suitable Lagrangian is

$$
2 \pi \int_{D^{d+1}} \omega
$$

or an integral multiple of the above. Firstly the above is independent of small fluctuations of the disk since $\omega$ is closed. Secondly, the above only depends on the boundary of the disk since $\omega$ is locally exact. Thirdly, there are two classes of disks one can choose. The ambiguity in the integrals (6.2) for the two choices is $2 \pi$ times an integer. Therefore the quantum mechanics is well defined when one puts (6.2) into the path integral.

The Cech cohomology arguments of the previous sections show that one can be less restrictive than the above. In other words, the cohomology argument is more general and it is always applicable. The homotopy argument can only be used in certain restrictive situations. It is not necessary to make assumptions about $\pi_{d}(M)$. We will construct some examples which have a non-trivial $\pi_{d}(M)$ yet there is charge quantization.

Consider the electromagnetic example of Sect. 2 but choose the trajectory to lie in the two torus $M=T^{2}$. In this case one has that $\pi_{1}\left(T^{2}\right)=\mathbb{Z} \oplus \mathbb{Z}$, but $\pi_{2}\left(T^{2}\right)=0$. The homotopy argument would not apply in this case and therefore one could not conclude anything about charge quantization. On the other hand, $H_{C}^{2}(M, \mathbb{Z})=\mathbb{Z}$ and we conclude that magnetic flux is quantized.

In a related paper [17], Witten used some homology arguments to work out the relationship homotopy spheres and the homology cycles required for the integration. This was required to determine the correct normalization for the topological Lagrangian. Since we are not worrying about normalization in this paper, we will not need such arguments.

Consider a two dimensional field theory with $M=S^{2}$. It is known that $\pi_{2}\left(S^{2}\right)=\mathbb{Z}$ and $\pi_{3}\left(S^{2}\right)=\mathbb{Z}$. The latter is responsible for the Hopf fibration $[7,13]$. The homotopy argument is not applicable in this case. The cohomology argument immediately tells us that there is no topological Lagrangian since the third cohomology class of any two dimensional manifold vanishes. Since $S^{2}=\mathrm{SO}(3) / \mathrm{SO}(2)$ it is clear that the such a non-linear sigma model would not contain a Wess-Zumino term. Consider the following non-linear sigma model:

$$
S^{2} \times S^{1}=(\mathrm{SO}(3) / \mathrm{SO}(2)) \times \mathrm{SO}(2) \text {. }
$$


The homotopy argument is not applicable yet the cohomology argument says there will be a quantization condition. It is interesting that there is no topological Lagrangian associated with either the $S^{2}$ or the $S^{1}$ but there is one associated with the product manifold $S^{2} \times S^{1}$.

In general there is no relationship between the cohomology groups and the homotopy groups. In certain cases there is a relationship between the homology groups and the homotopy groups, see p. 225 of [7].

Acknowledgements. I would like to thank my friends Dan Freed and I.M. Singer for their mathematics tutoring. I would especially like to thank Bruno Zumino for many discussions. I am indebted to the arm twisting of Michael Peskin, Shimon Yankielowicz and Bruno Zumino. Without their efforts this research have not been written up. My thanks to the referee for useful comments on improving the presentation of the material. I would like to thank the Alfred P. Sloan Foundation for the fellowship that has helped make this research possible.

\section{References}

1. Dirac, P.A.M.: Quantized singularities in the electromagnetic field, Proc. R. Soc. Lond. A133, 60 (1931)

2. Witten, E.: Global aspects of current algebra. Nucl. Phys. B223, 422 (1983)

3. Wess, J., Zumino, B.: Consequences of anomalous ward identities. Phys. Lett. 37B, 95 (1971)

4. Deser, S., Jackiw, R., Templeton, S.: Topologically massive gauge theories. Ann. Phys. 140, $372(1982)$

5. Deser, S., Jackiw, R., Templeton, S.: Three dimensional massive gauge theories. Phys. Rev. Lett. 48, 975 (1982)

Schoenfeld, J.: A mass term for three dimensional gauge fields. Nucl. Phys. B185, 157 (1981) Siegel, W.: Unextended superfields in extended supersymmetry. Nucl. Phys. B156, 135 (1979)

6. Bagger, J., Witten, E.: Quantization of Newton's constant in certain supergravity theories. Phys. Lett. 115B, 202 (1982)

7. Bott, R., Tu, L.: Differential forms in algebraic topology. Berlin, Heidelberg, New York: Springer 1982

8. Wu, T.T., Yang, C.N.: Dirac's monopole without strings: classical Lagrangian theory. Phys. Rev. D14, 437 (1976)

9. Feynman, R.P., Hibbs, A.R.: Quantum mechanics and path integrals. New York: McGraw-Hill 1965

10. Zumino, B.: Chiral anomalies and differential geometry. In: Relativity, groups and topology. II. DeWitt, B.S., Stora, R. eds. Amsterdam: North-Holland 1984

11. Singer, I.M., Thorpe, J.: Lecture notes on elementary topology and geometry. Berlin, Heidelberg, New York: Springer 1967

12. Misner, C., Thorne, K., Wheeler, J.A.: Gravitation. San Francisco, Freeman 1970

13. Eguchi, T., Gilkey, P.B., Hanson, A.J.: Gravitation, gauge theories and differential geometry. Phys. Rept. 66, 213 (1980)

14. Spanier, E.H.: Algebraic topology. New York: McGraw-Hill 1966

15. Nash, J., Sen, S.: Topology and geometry for physicists, London: Academic Press 1983

16. Rabinovici, E., Schwimmer, A., Yankielowicz, S.: Quantization in the presence of WessZumino Terms. WIS-84/30-PH

17. Witten, E.: Curent algebra, baryons, and quark confinement. Nucl. Phys. B223, 433 (1983)

Communicated by A. Jaffe

Received December 3, 1984; in revised form January 14, 1985 
